\title{
Implementation of Novel Group Delay Decomposition Method and Surgical Protocol for Assessing Peripheral Neuropathy
}

\author{
A Thesis \\ presented to \\ the Faculty of California Polytechnic State University \\ San Luis Obispo
}

\author{
In Partial Fulfillment \\ of the Requirements for the Degree \\ Master of Science in Biomedical Engineering
}

by Sammy Paul Nicolas

November 2012 
(C) 2012

Sammy Paul Nicolas

ALL RIGHTS RESERVED 


\section{COMMITTEE MEMBERSHIP}

TITLE:

AUTHOR:

DATE SUBMITTED:

COMMITTEE CHAIR:

COMMITTEE MEMBER:

COMMITTEE MEMBER:
Implementation of Novel Group Delay Decomposition Method and Surgical Protocol for Assessing Peripheral Neuropathy

Sammy Paul Nicolas

November 2012

Lily Laiho, Ph.D., Assistant Professor

Dan Walsh, Ph.D., Associate Professor 


\section{ABSTRACT \\ IMPLEMENTATION OF NOVEL GROUP DELAY DECOMPOSITION METHOD AND \\ SURGICAL PROTOCOL FOR ASSESSING PERIPHERAL NEUROPATHY \\ Sammy Paul Nicolas}

This paper outlines a surgical procedure for exposing and stimulating the sciatic nerve of an anesthetized rodent for purposes of obtaining conduction velocity readings. The ability to accurately quantify nerve conduction velocity has potential for use in the field of diagnostic medicine and disease characterization. An accurate reading depends on both the surgical method used to expose the desired nerve without imposing onto it any physical trauma as well as the stimulation protocol used to initiate action potentials. This paper contains the specific steps required to set up the necessary hardware and software for electrical stimulation as well as a detailed surgical and stimulation protocol. The animal model chosen to perform this experiment is the rat because it is the smallest animal model available with large enough nerve size to perform histology as a step toward validating the nerve size estimates obtained from this procedure. Based on repeated experimental runs, these methods are are expected to yield the most usable results while inflicting the least amount of physical trauma to the nerve. Upon isolating the sciatic nerve, the surgeon is to place an electrode cuff around the nerve and then initiate the stimulation protocol. The stimulation software is designed to slowly increment the current passing through the nerve to recruit increasing numbers of neurons one-at-a-time. The resulting data would theoretically offer researchers the actual threshold values for each individual neuron, uncovering information about the conduction characteristics of each one. The steps outlined in this thesis have been optimized to implement the theoretical model of group delay. Using the decomposition model introduced by Szlavik et al., the signal obtained from the entire nerve is broken down into individual action potentials associated with individual neurons.

Keywords: neuron, action potential, group delay, electrical stimulation 


\section{ACKNOWLEDGEMENTS}

I would like to dearly thank my family, friends and colleagues who have offered me nothing but support and encouragement throughout my research. Out of all those who believed in me, I feel there are a few who deserve special thanks. I am deeply grateful for the support and guidance of Dr. Robert Szlavik throughout the entire project. His boundless encouragement was a reminder that there was always someone rooting for me. His gift of communication and particularly his patience made this a tremendously valuable learning experience for me, and it is this for which I am most grateful. I will never forget this. Thank you, Dr. Szlavik, for everything.

My parents, Paul and Nina, have always been willing to lend a sympathetic ear through the most trying times, and have been my rock throughout this undertaking. Never have you wavered in your support and love. For this and for so much more, I am eternally grateful and lucky to have you both as wonderful parents. Thanks, Mom and Dad.

My brother and sister have had nothing but praise for my efforts throughout this time. At times through this experience I have had to be absent from family events, both big and small. Their understanding and love reminded me what family is for. Natalie, your knowledge of physics and science inspired me to always aim high to achieve results. Chris, your masterful social abilities and humble compassion for others taught me the reward of having faith in people. I love you guys and you know I could not have done this without you.

Last, but not least, I would like to thank my research partner Christopher Blake Finnegan. As my research partner, he has been there every step of the way from the the endless hours in lab to the endless hours behind the computer. Thank you, Blake. 


\section{TABLE OF CONTENTS}

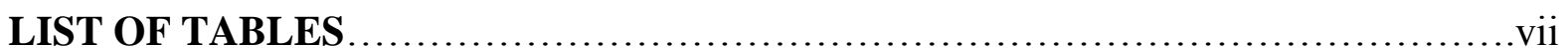

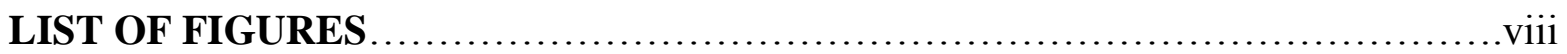

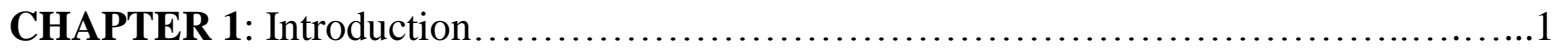

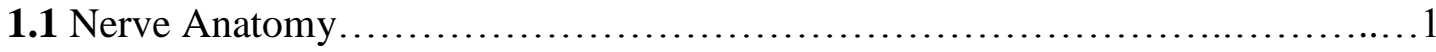

1.2 Overview of the Action Potential............................................... 3

1.3 Threshold Responses......................................................

CHAPTER 2: The Nernst Potential................................................. 7

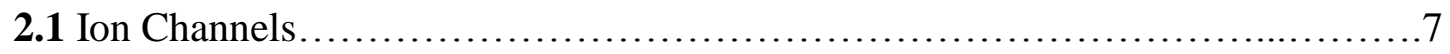

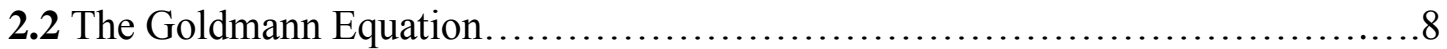

CHAPTER 3: Action Potential Anatomy …....................................... 10

CHAPTER 4: Group Delay........................................................... 13

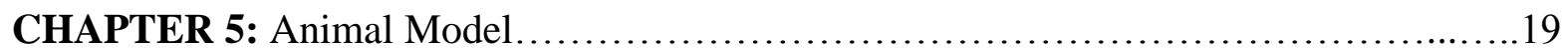

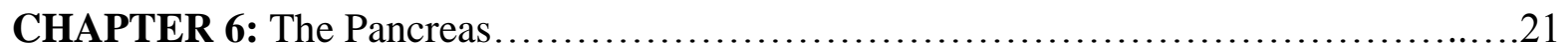

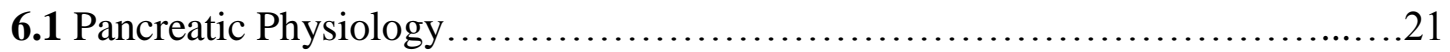

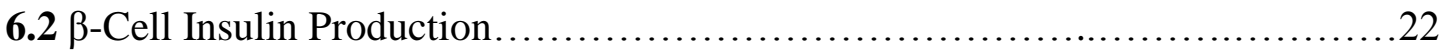

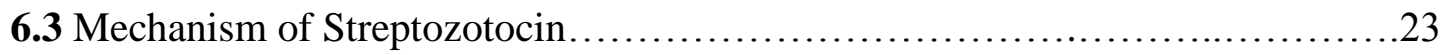

CHAPTER 7: Materials and Laboratory Fixtures......................................25

CHAPTER 8: Methods............................................................. 40

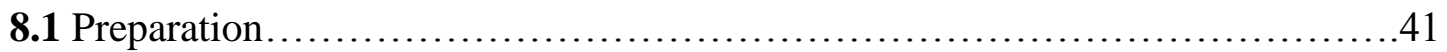

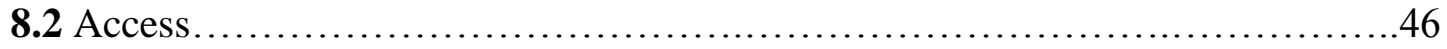

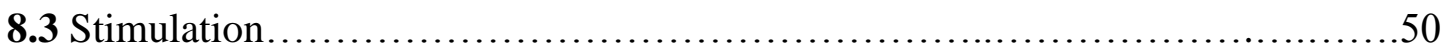

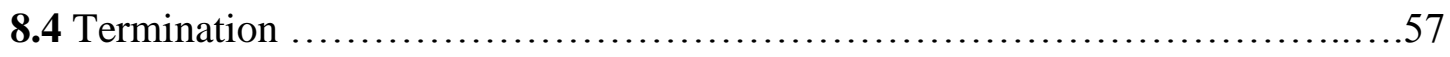

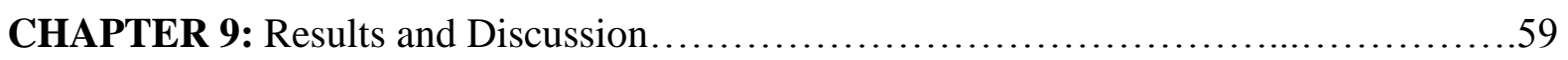

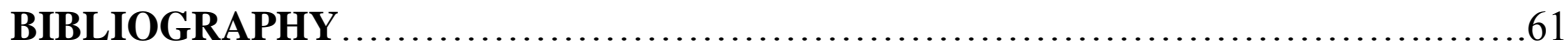




\section{LIST OF TABLES}

TABLE 1: List of Symbols.................................................. 16

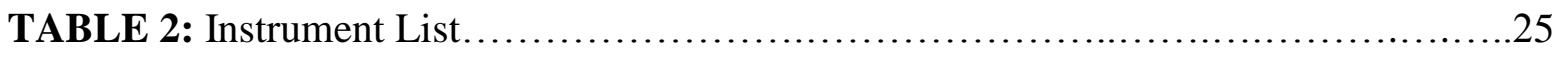

TABLE 3: Laboratory Fixtures............................................. 32

TABLE 4: Stage 1 Parameter Values...........................................51

TABLE 5: Stage 2 Stimulator Values......................................... 53

TABLE 6: Differential Amplifier Settings............................................54 


\section{LIST OF FIGURES}

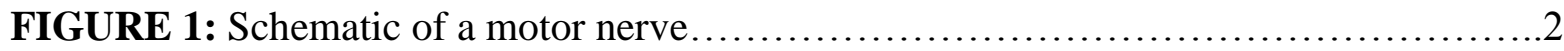

FIGURE 2: Subthreshold / Suprathreshold Waveforms...............................

FIGURE 3: Ionic Channel Activity.................................................... 8

FIGURE 4: Action Potential Waveform......................................... 10

FIGURE 5: Three-Electrode Schematic..............................................

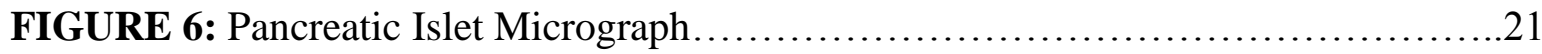

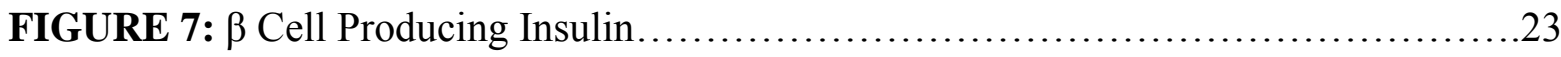

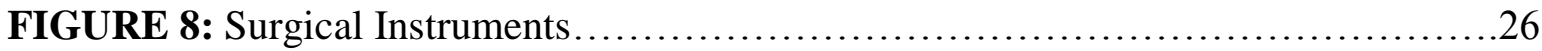

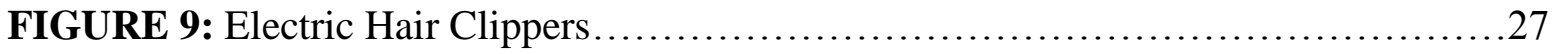

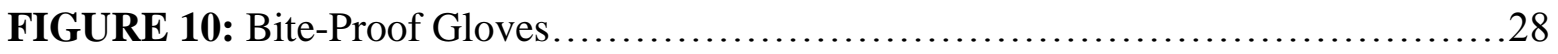

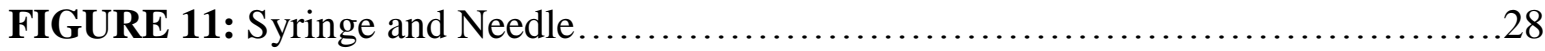

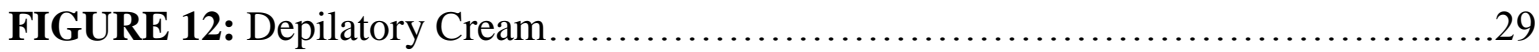

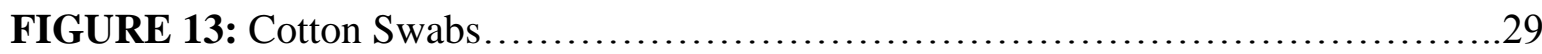

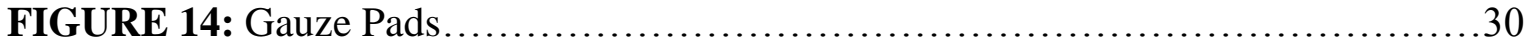

FIGURE 15: Laboratory Gloves..................................................

FIGURE 16: Sharps Container....................................................

FIGURE 17: Nose Cone .......................................................... 31

FIGURE 18: Differential Amplifier.................................................33

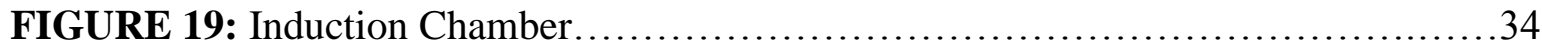

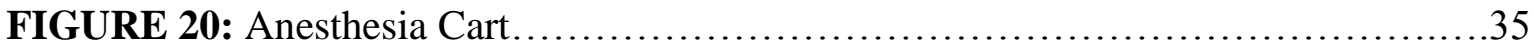

FIGURE 21: Oscilloscope .........................................................

FIGURE 22: Electrode Cuff............................................................

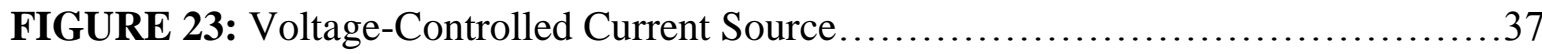

FIGURE 24: Electrode Adapters.......................................................

FIGURE 25: Level Indicator on Anesthesia Cart....................................42

FIGURE 26: Threaded Connectors................................................

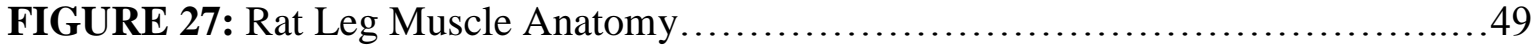

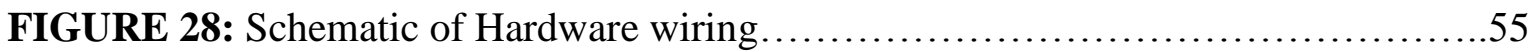




\section{Chapter 1}

\section{Introduction}

The human nervous system is a complicated network of neurons whose purpose is to receive and pass signals to affect the activation of muscle tissue and to relay sensory information of stimuli present in the environment. A peripheral nerve represents the integration of complex systems of cells, proteins, ionic balances, and many other properties that contribute to its individual ability to carry and generate signals. It is these measurable, quantitative characteristics that allow for study of the behavior of the healthy nerve as well as those of the disease model.

Afflictions such as Type 1 Diabetes Mellitus and Chronic Inflammatory Demyelinating Polyneuropathy (CIDP) are known disrupters of normal neural function. These, as well as other neuropathies, affect various neural targets in different ways. The disruption brought on by these diseases and many others is quantified in clinical settings not only by differences in the voltage threshold, but also the time an action potential takes to travel a fixed distance [2]. Thus, it is important to assess the symptoms of these diseases using a standardized, reproducible test protocol. Electrophysiology-based tests allow clinicians to assess the conduction behavior of nerves in response to electrical stimulation. In such tests, parameters such as those mentioned above are measured and used to diagnose neuropathy.

\subsection{Nerve Anatomy}

The nervous system is comprised of sensory and motor nerves. Generally, a nerve is comprised of elongated conductive cells called neurons, in bundles. These bundles are held together by neuroglia, cellular bodies which provide structural framework and assist with 
insulation and signal conduction. Sensory nerves, as their name suggests, are responsible for transmitting information from the environment to the central nervous system for signal processing. Motor nerves, alternatively, transmit signals distally from the central nervous system to the periphery, initiating skeletal muscle contractions. A schematic of a motor neuron is indicated below, with major regions highlighted in Figure (1).

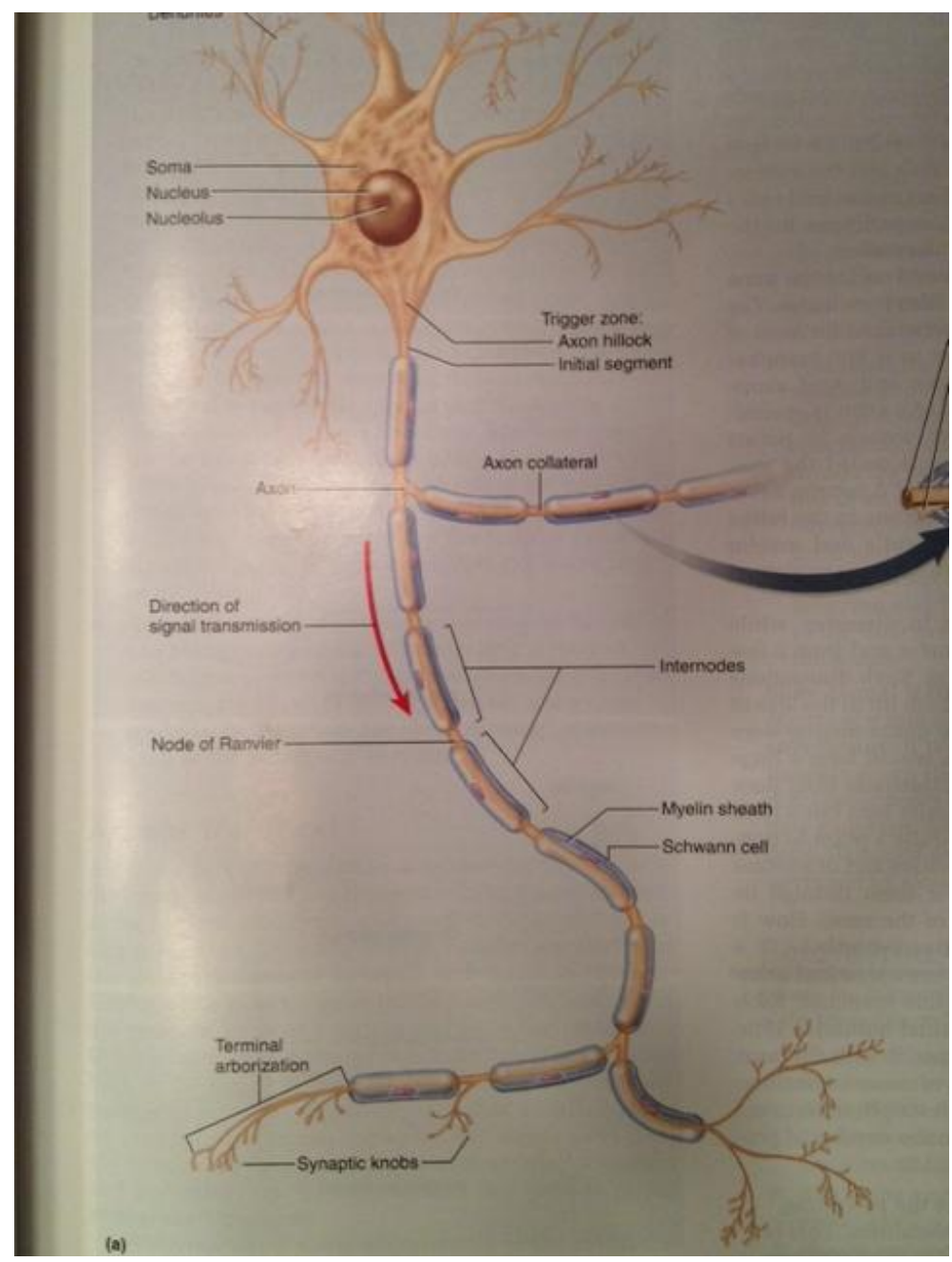

Figure 1: Schematic of a motor nerve [1].

A neuron's anatomy reflects its function. Beginning at the location of the neuron which receives the signal first, the dendrites form the most proximal section of the neuron and function as receptors that receive input signals from a preceding neuron. Branched in 
shape, these dendrites are able to receive signals from multiple locations and transmit them down the neuron. This branched section then conveys the signal to the soma, also called the cell body. The soma houses two especially important neural microstructures which are vital for survival: the nucleus and the axon hillock [1]. The nucleus produces and transports all of the proteins required by the neuron for repair, day-to-day maintenance and production of enzymes and signaling ions. The axon hillock - the triangular structure between the axon and the soma-is the starting point of the axon. It is often the site where action potentials are generated, due to the high concentration of voltage-gated ion channels present there. From the soma, the signal is then passed through the axon hillock along the length of the entire axon until it reaches its final destination, the axon terminal. Upon reaching axon terminal, the action potential causes the presynaptic neuron to release chemical signals, in the form of neurotransmitters, across a synaptic gap [1]. These neurotransmitters ultimately either excite the postsynaptic neuron or inhibit it. It is these electrical signals passed between neurons, all over the body, that form the basis of information transmission and processing.

\subsection{Overview of the Action Potential}

The action potential is the name given to the change in membrane potential travelling down the neuron. In a healthy human, an initial action depolarization in efferent nerves causes a cascade of neurons to fire similarly in sequence until the action potential reaches the neuromuscular junction. Once this threshold is reached, complete depolarization occurs and the electrical signal is passed forward. The signal travelling down the axon is essentially a series of depolarizations propagating down the nerve wherein the membrane polarity is temporarily changed, with the potential inside the neuron becoming temporarily positive relative to the extracellular potential which is the reference. Membrane polarity will be 
discussed in detail later in this section. This depolarized region propagates down the axon in the downstream direction, referred to as orthodromic conduction [1]. This occurs as a result of a physiological phenomenon called the cell's refractory period. Despite the tendency of the wave of polarity to propagate in both directions, immediately following the depolarization is the absolute refractory period which prevents against further stimulation for a short time. Immediately after an action potential occurs, there is a period of time during which another action potential cannot be caused, regardless of stimulus amplitude. Were it not for this time period in which a cell cannot recruit enough $\mathrm{Na}^{+}$channels to cause another action potential, neurons would be susceptible to stimulation of too high a frequency. That is, the absolute refractory period limits the rate at which a neuron can fire. Thus, under normal conditions the depolarization propagates down the axon away from the soma, and acts as a frequency limiter to prohibit stimulation overload. It is notable that during the absolute refractory period, no additional action potentials can be initiated, no matter what the stimulus amplitude.

\subsection{Threshold Responses}

The threshold is the minimum voltage at which an action potential is triggered. This threshold functions such that any voltage smaller than the threshold does not cause an action potential; and a stimulation equal to or greater than the threshold does cause one. A subthreshold current injection does not necessarily imply that simply nothing happens. Subthreshold current injection results in a potential response that is linear in the sense that the membrane behaves as a paralleled RC circuit. The nonlinear sodium channel effect, specific to an action potential, comes in as the stimulus reaches a point such that the 
depolarization is near threshold. In this case, a slight change in membrane potential is seen as illustrated in Figure (2) below:

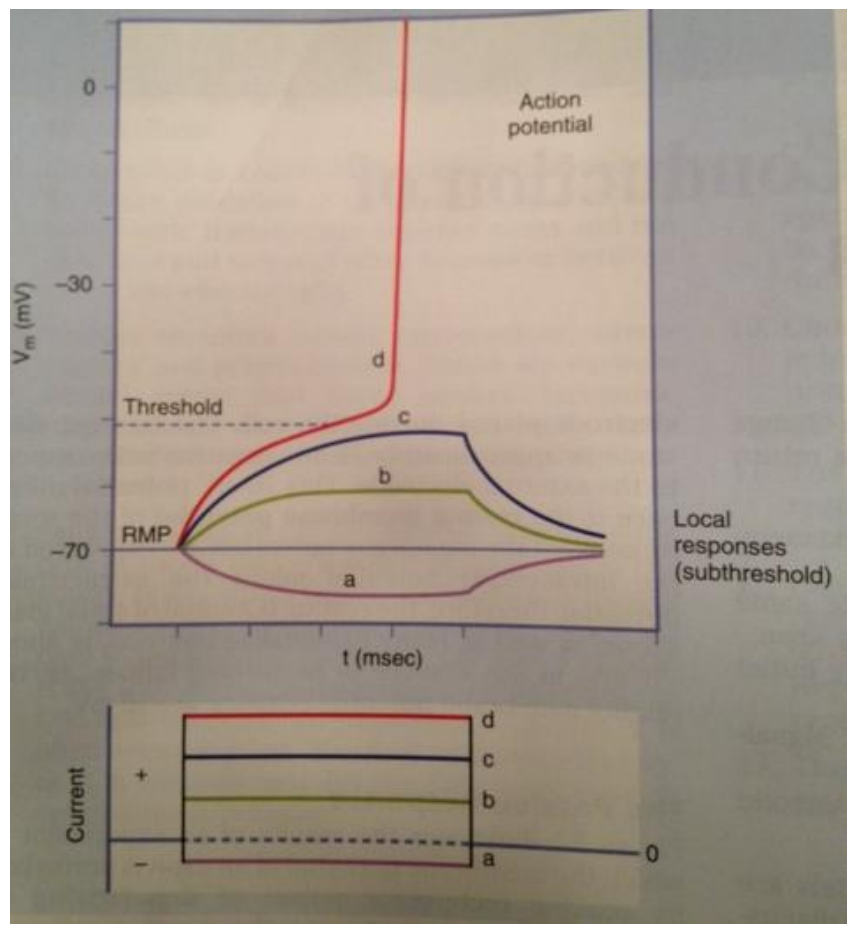

Figure 2: Results b, c are from a subthreshold stimulus pulse; a hyperpolarizing pulse; $d$ suprathreshold pulse [1].

A suprathreshold response, on the other hand, causes a spike in membrane potential. This represents a short-lived yet significant change in electric potential from that associated with the negative resting membrane potential (RMP). During this phenomenon, the membrane potential can spike up from $-70 \mathrm{mV}$ to greater than $+50 \mathrm{mV}$ in the case of some mammalian motor neurons [1].

Some mammalian motor neurons have an inherent voltage differential of approximately $-70 \mathrm{mV}$, called the resting membrane potential (RMP). This resting membrane potential, by convention, is the steady-state potential difference of the intracellular region subtracted from the extracellular region. This resting membrane potential difference occurs as a result of the concentration differences and conductances of the different ionic species 
present both inside and outside the cell, particularly $\mathrm{Na}^{+}, \mathrm{K}^{+}$, and $\mathrm{Cl}^{-}$. The potential difference of the cell at equilibrium is also referred to as the Nernst Potential. 


\section{Chapter 2}

\section{The Nernst Potential}

The Nernst Potential seen below in equation (1) is named after Walther Nernst, the German chemist and physicist who developed the equation used to calculate the potential difference across an ion-permeable membrane given specific ion concentrations. It is related to the RMP, with the notable exception that instead of using all membrane-permeable ionic species, it uses only one. In this expression, $R=8.831451 \mathrm{~J} \mathrm{~K}^{-1} \mathrm{~mol}^{-1}, T$ is the absolute temperature in Kelvin, $z$ is the ionic valence for a single ionic species $N$, and $F=96485{ }^{\circ} \mathrm{C}$ $\mathrm{mol}^{-1}$.

$$
E_{N}=\frac{R T}{z F} \ln \frac{[N]_{o}}{[N]_{i}}
$$

\subsection{Ion Channels}

The cellular membranes are semipermeable to these different ionic species, and out of those species to which the membranes are permeable, some exert a greater influence on the membrane potential than others. In a hypothetical cell which is permeable to only one ion, the RMP will equal the membrane potential of this ion. In real animal cells, which are permeable to numerous ions, the RMP is related not only to their respective ionic potentials and relative ionic concentrations, but also to their relative permeabilities. The greater the permeability a membrane has to an ion, the greater the influence it has on the RMP of the

cell. The permeability of the membrane to a particular ion is a factor of both the number of available ionic channels through which the ion can pass during depolarization and the ease with which the ion can pass through each channel. Ion channels are proteins present on the 
surface of the cell which allow certain ions to pass through them while blocking others as shown in Figure (3):

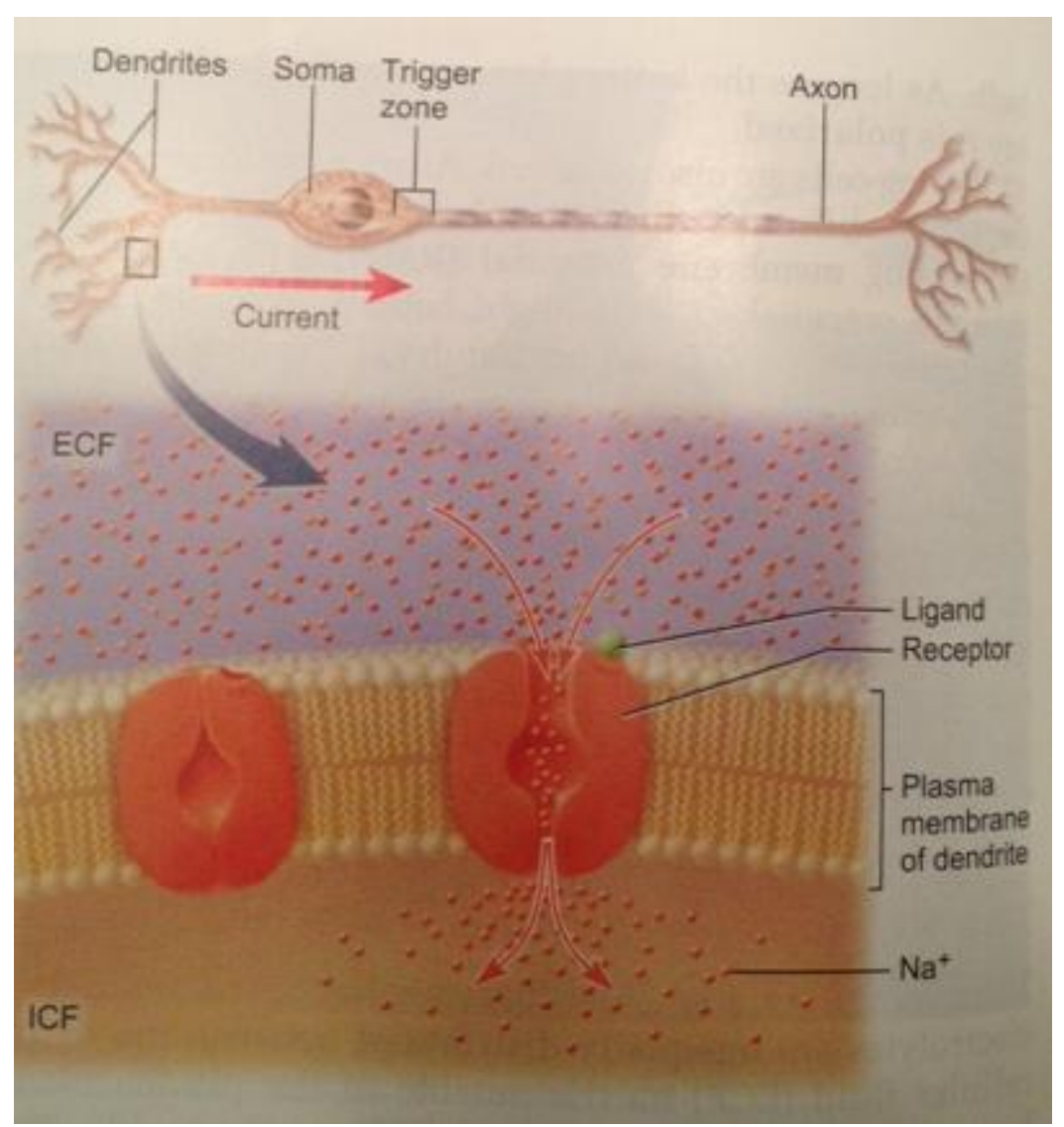

Figure 3: Ionic Channel Activity [1]

In a situation where the membrane potential does not equal the equilibrium potential, ions tend to move so as to bring the potential to the equilibrium potential for that ion. Depending on such factors as the direction the ion is moving relative to its gradient and the species of ion itself, the driving force behind equilibrium potential can be electrochemical force or a concentration gradient.

\subsection{The Goldmann Equation}

To determine the RMP for a membrane that is permeable to two or more ions, the Goldmann Equation can be of use: 


$$
E_{m}=58 m V \log _{10}\left(\frac{\left[K^{+}\right]_{o}+b\left[N a^{+}\right]_{o}+c\left[C l^{-}\right]_{o}}{\left[K^{+}\right]_{i}+b\left[N a^{+}\right]_{i}+c\left[C l^{-}\right]_{i}}\right) \quad b=\frac{p_{N a}}{p_{K}}, c=\frac{p_{C l}}{p_{K}}
$$

In the above equation, $E_{m}$ is defined as the transmembrane potential. $58 \mathrm{mV}$ is used as the multiplier because of the assumption of room temperature. The values $p_{N a}, p_{K}, p_{C l}$ are the relative permeabilities of the ions $\mathrm{Na}, \mathrm{K}$, and $\mathrm{Cl}$ respectively. This equation accounts for the different ionic species that influence the RMP based not only on concentration, but also on specific ionic permeability. 


\section{Chapter 3}

\section{Action Potential Anatomy}

As stated above, the action is the name given to the signal transmitted along neurons for the purpose of stimulating or inhibiting subsequent neurons. The waveform associated with an action potential can be broken down into different sections, each defined by specific ionic events taking place within each neuron. An example of an action potential is illustrated by the graph below in Figure (4):

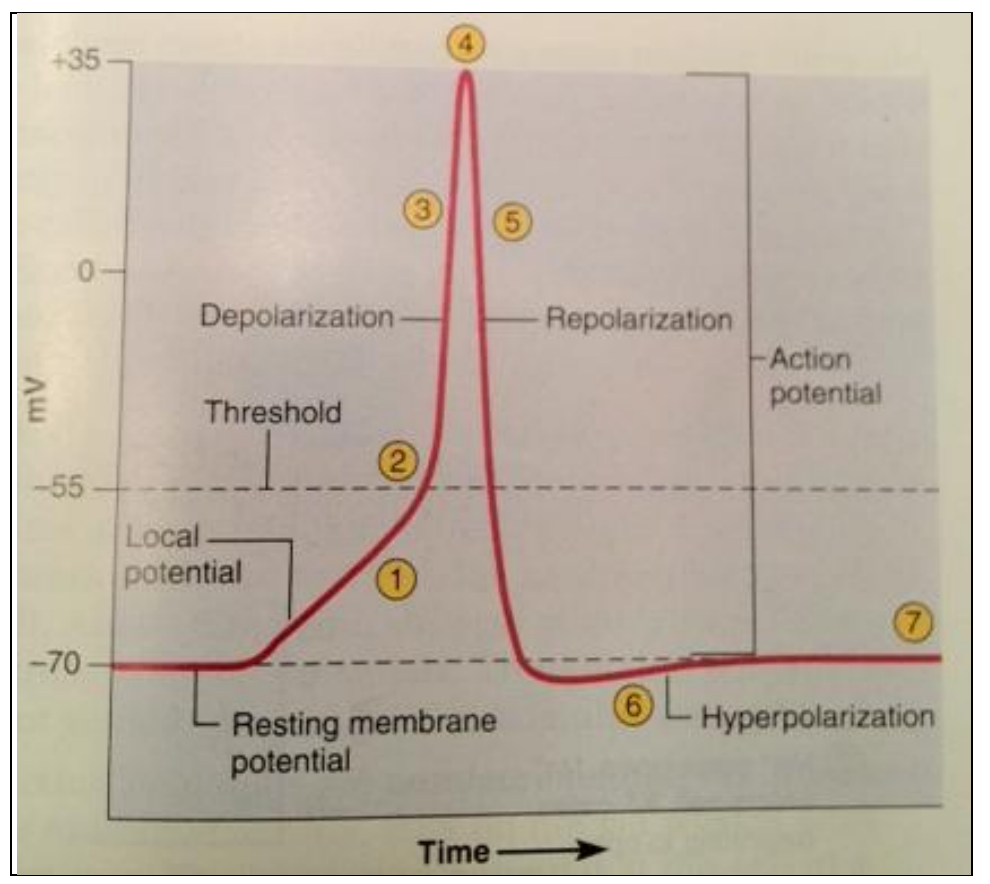

Figure 4: The illustration of the different stages of an action potential from RMP to hyperpolarization [1].

One will notice the initial spike from intracellular RMP to a temporary positive value nearing $+40 \mathrm{mV}$. In some neural cells, this peak value can be as high as $+50 \mathrm{mV}$. It can be attributed to the initial opening of $\mathrm{Na}^{+}$ion gates in response to the depolarization and thus reflects the influx of $\mathrm{Na}^{+}$into the cell, which further depolarizes the cell. The next phase is a combination of the decrease of $\mathrm{Na}^{+}$conductance and an increase in $\mathrm{K}^{+}$conductance, both of 
which act together to ultimately repolarize the cell. However, there is a portion of the action potential that dips past the RMP resulting in hyperpolarization, called the undershoot. The $\mathrm{K}^{+}$ gates are responsible for this phenomenon because these gates do not close immediately after depolarization and therefore remain open long enough to keep on repolarizing the cell until the membrane potential approaches that of $\mathrm{K}^{+}$. By the time the membrane potential reaches RMP again, the $\mathrm{K}^{+}$gates have fully closed and the refractory period is over, allowing for another depolarization. The action potential graph in Figure (4) above also illustrates both the absolute refractory and relative refractory periods. From the time of initial depolarization until the repolarization nearly reaches RMP, an action potential cannot be caused. This is due to the fact that most of the $\mathrm{Na}^{+}$channels are inactivated and thus cannot be stimulated until after repolarization. After this period comes the relatively refractory period during which the cell can be made to fire another action potential, but a higher-than-normal voltage stimulus is required to do so. At this point, the higher-than-normal voltage stimulus required is used to compensate for both the decreased number of $\mathrm{Na}^{+}$gates available and the elevated conductance of $\mathrm{K}^{+}$still present from the initial depolarization.

The action potential response is not constant throughout the entire axon of a nerve. Many nerves of the peripheral nervous system are wrapped by the plasma membranes of Schwann cells, called myelin sheaths, which insulate the nerve fiber. Effects of this form of insulation are apparent in conduction velocity. These myelin sheaths can be seen at regular intervals along the axon and are separated by bare sections of nerve called Nodes of Ranvier. The effect that is attributed to this property is an overall increase in conduction velocity from soma to axon terminal. From an equivalent circuit perspective, the substantial increase in 
conduction velocity is predominantly due to a decrease in membrane capacitance attributed to the presence of the myelin sheath.

The myelin also limits the amount of propagation of action potentials to the effect that it does not require that a new action potential be initiated in myelinated sections of nerve fiber [3]. The action potential is regenerated at each Node of Ranvier and the conduction of the action potential is electrotonic under the myelin such that when an action potential reaches a Node of Ranvier there is sufficient potential at the next Node of Ranvier to regenerate the next action potential. This property is called saltatory conduction. Under this property, action potentials are generated only at each Node of Ranvier, and travel relatively quickly between them through the myelinated sections, instead of having to constantly regenerate [3]. 


\section{Chapter 4}

\section{Group Delay}

Patients afflicted by Type 1 Diabetes Mellitus often experience symptoms across multiple body systems. From retinopathy to heart problems to numbness in the foot, there is a multitude of potential targets for the disease. Most commonly affected areas include the cardiovascular system, the organ systems, and the nervous system. The nervous system, in particular, presents a unique challenge for noninvasive measurement of nerve viability due to the delicate nature of nerves as well as the challenge to obtain accurate information noninvasively. The current standard is the nerve conduction velocity test. This simple electronic assessment method involves a clinician placing cutaneous electrodes onto the patient's appendage of interest and applying a current to measure the conduction velocity of the entire nerve trunk. This process returns a scalar value to the user. This value will either fall within the normal range or outside the range, correlating roughly to the level to which the disease has progressed. Assessment, then, relies on the difference between the patient's conduction velocity and an average acceptable nerve conduction velocity. This technique has many desirable characteristics including low cost, noninvasiveness, and time-tested results. Limitations, however, do exist. Notably, no information about the underlying neurons contributing to the signal is given. While the nerve trunk conduction velocity can potentially be of use in diagnostics, it would be even more beneficial to retrieve the conduction velocities of the individual neurons comprising the overall signal. That way, assessment targets would include individual neurons, which would provide more detailed information than a single nerve trunk measurement. 
Such a practice would require isolation and stimulation of individual nerve fibers in a living patient to be carried out properly. This most certainly implies neurosurgery, which is not cost effective in most cases. Because this is not practically feasible in most diagnostic settings, a technique has been developed by Szlavik et al. [4] that circumvents those challenges. The technique proposes a 3-electrode setup consisting of a single stimulation electrode and two recording electrodes which record the compound evoked potential.

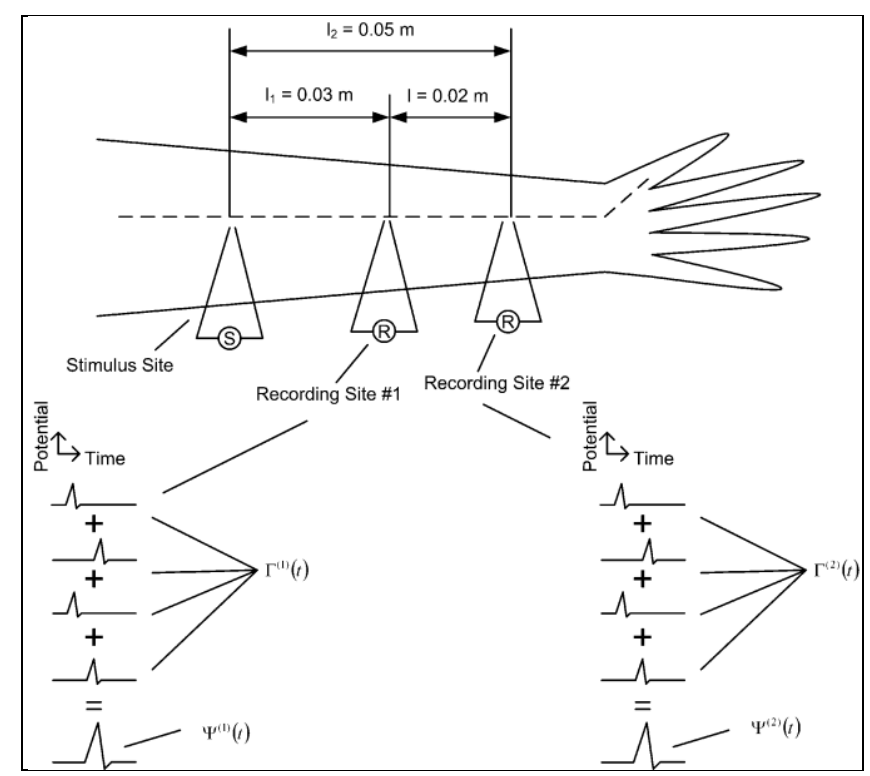

Figure 5: Three Electrode Schematic [4]

Incrementally stepping up the stimulation current using small enough steps will, in theory, recruit more and more nerve fibers until maximum conduction is reached. With each additional fiber recruited, the output signal increases in magnitude. As the increment steps become smaller and smaller, fewer and fewer additional fibers will be recruited with each subsequent step until they become so small that only one new fiber gets recruited with each new step. Then, subtracting the compound evoked potential from the previous one will yield the potential associated with a single fiber. This is the foundation of the group delay approach. 
Of note are several assumptions of particular importance when performing this experiment. First, the time delay - the time it takes for the signal to travel from the stimulator to each fiber plus the time it takes each fiber to respond — at the stimulation site is negligible. Second, a constant threshold value is assumed as well, though this can fluctuate over a small range in real fibers. Third, it is assumed that the step resolution of the hardware is small enough to ensure that an increase in compound evoked potential is solely the result of a single additional nerve fiber firing. This is a necessary assumption because the entire theory is premised on the ability to isolate the signal associated with a single nerve fiber. A linear relationship is assumed between conduction velocity and fiber diameter. This simplifying assumption essentially limits the scope to a healthy model, because demyelinating neuropathies can cause the relationship between fiber diameter and conduction velocity to become nonlinear, which is beyond the scope of this technique. Table (1) given below gives the variable names used to identify the quantities used in the theoretical model of the group delay approach. 
Table 1: List of Symbols

Variable

\begin{tabular}{|cl|}
\hline$d$ & Fiber Diameter \\
$\eta$ & Constant: $3.5 \times 10^{5} \mathrm{~m}^{-1}$ \\
$\zeta$ & Current Constant $(10 \mathrm{~mA})$ \\
$\xi(\mathrm{d})$ & Activation Function \\
$i$ & Current Step \\
$\Psi^{\mathrm{n}}(\mathrm{t})$ & Compound Evoked Potential \\
$\mathrm{n}=1,2$ & Recording Sites \\
$\Omega_{i}$ & Current Amplitude \\
$d_{k}$ & Diameter of $k$ th Fiber \\
$\overline{\boldsymbol{r}}$ & Perpendicular Depth Between Recording \\
$u$ & Site and Center of the $k$ th Fiber \\
$u$ & Positive Step Function \\
$\mathrm{G}$ & Single Fiber Action Potential \\
$v_{k}$ & Conduction velocity of $k$ th fiber \\
$\delta_{\mathrm{k}}$ & Propagation Delay of SFAP \\
$\boldsymbol{\Gamma}_{\boldsymbol{i}-\mathbf{1}}^{(\boldsymbol{n})}(\boldsymbol{t})$ & SFAP Waveform \\
$\boldsymbol{H}_{\boldsymbol{i}-\mathbf{1}}(\boldsymbol{f})$ & Frequency Response \\
$\boldsymbol{\tau}_{\mathbf{i}-\mathbf{1}}$ & Group Delay \\
$\theta$ & Phase Response \\
\hline
\end{tabular}

These assumptions in hand, the first task is to adopt a function to determine whether or not given current amplitude is sufficient to excite each fiber of diameter $d$ :

$$
\xi(d)=\zeta \exp [-\eta d]
$$

Equation (3) above uses the product of the fiber diameter $d$ and constant $\eta=3.5 \times 10^{5} \mathrm{~m}^{-1}$. This exponential multiplied by a constant current $\zeta=10 \mathrm{~mA}$ yields the activation function $\xi(\mathrm{d})$. As it is the compound potential that is of concern, an equation to compute the compound potential for each current step $i$ is given in (4):

$$
\Psi_{i}^{(n)}=\sum_{k=1}^{m} u\left[\Omega_{i}-\xi\left(d_{k}\right)\right] \cdot G\left[v_{k} \cdot\left(t-\delta_{k}^{(n)}\right), \bar{r}\right]
$$

Equation (4) above gives $\Psi$, the compound evoked potential at each recording site $n=1,2$. Current amplitude at each step $i$ is represented by $\Omega_{i} ; d_{k}$ is the diameter of the $k$ th fiber and $r$ 
is the perpendicular depth between the recording site and the center of the $k$ th fiber. The single fiber action potential is defined by $\mathrm{G}\left[v_{k} \cdot\left(t-\delta_{k}{ }^{(n)}\right), \bar{r}\right]$ comprising $\Psi$, with positive step function $u ; v_{k}$ is the conduction velocity of the $k$ th fiber and $\delta_{\mathrm{k}}$ is the propagation delay of the single fiber action potential (SFAP) between the two recording sites. The compound evoked potential is the sum of the single fiber action potentials at any time $t$. The compound evoked potentials are computed for each current step $\Omega_{i}$ at each recording site $\Psi^{1}(\mathrm{t})$ and $\Psi^{2}(\mathrm{t})$. Waveforms are then extracted from these compound evoked potentials by subtracting each compound evoked potential from the preceding one as per Equation (5):

$$
\Gamma_{\mathrm{i}-1}^{(\mathrm{n})}(\mathrm{t})=\Psi_{\mathrm{i}}^{(\mathrm{n})}(\mathrm{t})-\Psi_{\mathrm{i}-1}^{(\mathrm{n})}(\mathrm{t}) \quad \text { for } 2 \leq \mathrm{i} \leq \mathrm{q}+1
$$

The resulting SFAP waveforms $\Gamma_{i-1}^{(n)}(t)$ will then represent the single fiber action potential. In cases where no additional nerve fiber was recruited, the signals will be of the same magnitude before and after the current is stepped up, resulting in $\Gamma_{i-1}^{(n)}(t)=0$. As per the assumptions mentioned above, if the current resolution is not high enough, $\Gamma_{i-1}^{(n)}(t)$ will represent the action potentials associated with more than one fiber.

The actual group delay is then found using the ratio of the Fourier transforms of two subsequent SFAPs between the proximal and distal recording sites. In the time domain, the product of the input signal and the impulse response is equal to the output. Simple algebraic rearrangement of the equation yields the impulse response as the ratio of the output divided by the input signals. This principle is applied here by taking the ratio of the Fourier transform of the SFAP of the distal site $(n=2)$ divided by that of the proximal site $(n=1)$, shown below in Equation (6): 


$$
H_{i-1}(f)=\frac{F\left[\Gamma_{i-1}^{(2)}\right]}{F\left[\Gamma_{i-1}^{(1)}\right]}
$$

All terms in the above equation are expressed in the frequency domain via the Fourier Transform, denoted by $F . H_{i-1}(f)$ represents the frequency response, $\Gamma_{i-1}{ }^{(2)}$ represents the SFAP at the distal recording site, and $\Gamma_{i-1}^{(1)}$ represents the SFAP at the proximal recording site. The Fourier Transform of the SFAP expresses the signal in the frequency domain, instead of the time domain. This gives the frequency response of a given fiber. Ultimately, this can be used to find the group delay, shown below:

$$
\tau_{i-1}=\left(\frac{-1}{2 \pi}\right)\left(\frac{d \theta_{i-1}(f)}{d f}\right)
$$

The rate at which the phase response changes with respect to frequency gives the group delay, which is the transit time of the signal between the two recording sites. 


\section{Chapter 5}

\section{Animal Model}

The animal model chosen for this study is the rat. Since the Institutional Animal Care and Use Committee (IACUC) regulations dictate that animal studies must be carried out on the lowest-order animal model allowable, the rat is justified on both a neurological and anatomical basis. The neural anatomy of the rat is comparable to that of the human, as is the function and physiology of the nervous system [7].

Due to the delicate nature of this neurological study - in particular the very limited physical space constraints the surgeon encounters when attempting to expose such a small segment of nerve - it is important to use an animal model that is easy to house and handle. The advantage of using a small animal model for this experiment is that, in accordance with IACUC regulations, housing is manageable, and relatively undemanding compared to the housing requirements of larger animals. Regarding the procedure itself, the rat's small size allows for simple manipulation and stabilization of the hindlimb in the steps preceding nerve exposure. It is important, however, not to use an animal model that is too small. The animal must have large enough peripheral nerves to allow for reproducible experimentation without excessive variability from subject-to-subject. And once the nerve is fully isolated from surrounding tissue, there must be sufficient space surrounding it such that the surgeon can consistently place the electrode around the same segment of nerve in all experimental trials. That is, upon exposing the desired nerve segment, there must be enough physical space around it to set the electrodes in the same location consistently from animal-to-animal. 
Fulfilling the aforementioned considerations, the rat was chosen as the optimum animal model for the following reasons:

- The rat is neurologically comparable to humans both anatomically and physiologically

- The rat is of relatively small size and easily handled

- The space around the sciatic nerve allows for ease of electrode placement

- The rat's large sciatic nerve makes it simple to obtain sufficient distribution of nerve fiber diameters 


\section{Chapter 6}

\section{The Pancreas}

The pancreas is an elongated spongy organ located below and behind the stomach, performing both endocrine and exocrine functions during digestion. The endocrine pancreas is primarily responsible for secreting insulin during and immediately after meal ingestion. Scattered throughout the pancreas are pancreatic islets, which make up roughly $2 \%$ of the organ. A micrograph of a pancreatic islet cell is shown below in Figure (6). It is obvious by the variety of cell morphology visible in the figure below that an islet consists of numerous types of cells.

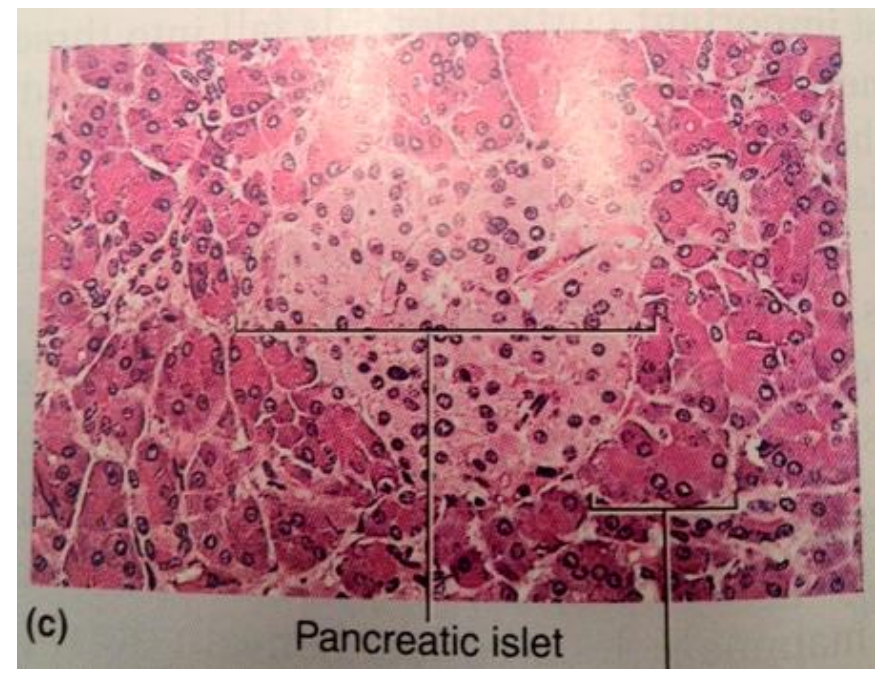

Figure 6: A micrograph of a pancreatic islet. The different morphologies of cells visible show $\alpha, \beta$, and $\delta$ cells [3].

This small portion of the pancreas, however, is vitally important for glycemic regulation. These islets contain several cell types, but of particular significance are the $\beta$ cells.

\subsection{Pancreatic Physiology}

The insulin produced by the pancreas is secreted exclusively through $\beta$ cells in response to detection of food ingestion. Food contains glucose to nourish the body and 
insulin acts as a stimulator for cells to absorb it from the bloodstream. In addition to facilitating in glucose uptake, insulin also promotes glucose storage in the muscle and adipose tissue, which lessens the burden of glucose output on the liver. This absorption process lowers the amount of the glucose in the bloodstream, known as the blood glucose level. Hyperglycemia, or excessive glucose in the blood, is a sign of diabetes and acts as evidence that insufficient levels of glucose are being absorbed from the blood to be used in the rest of the body [3].

In diabetic patients, the action of insulin is partially or completely inhibited by the disease. But the patient's cells still require nutrient nourishment for survival. So the cells retrieve these necessary nutrients from other sources. In untreated cases the body resorts to metabolizing fat and protein. If this is allowed to go untreated, with time, it results in severe muscle atrophy and emaciation.

\section{2 $\beta$ Cell Insulin Production}

The $\beta$ cell of pancreatic islets follows a specific pathway governing the production of insulin, shown below in Figure (7). 


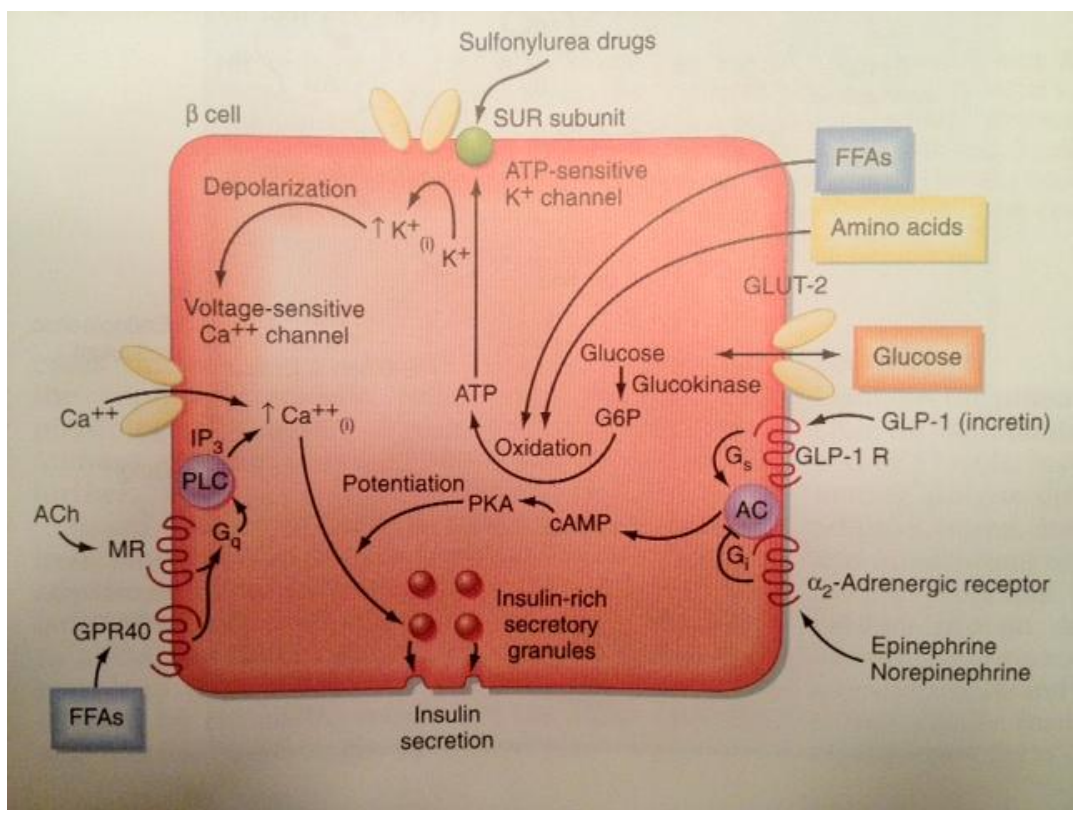

Figure 7: A $\beta$ cell using Glucose to produce insulin [1].

Glucose detection is the primary stimulator for insulin production. The GLUT2 transporter facilitates the entry of glucose molecules into $\beta$ cells, where the glucose is phosphorylated to G6P by glucokinase. The glucokinase also controls the rate of glucose entry into the cell by correlating it to the rate of glucose phosphorylation. This process increases the ratio of ATP to ADP within the cell, which closes the ATP-sensitive $\mathrm{K}^{+}$channel. When this channel closes, the $\beta$ cell membrane depolarizes, opening the voltage-gated $\mathrm{Ca}^{++}$channels, causing increased levels of calcium within the cell. Increased calcium within the cell then activates exocytosis of insulin.

\section{3: Mechanism of Streptozotocin}

Type 1 Diabetes Mellitus was induced in the rodent by a single intraperitoneal (IP) injection of Streptozotocin (STZ). Injection was required because the healthy rat model used for this experiment does not develop the disease spontaneously, nor does it develop diabetes from a Western diet alone. Instead, the STZ injection targets the $\beta$ cells of the pancreas [5]. The $\beta$ cells are responsible for producing insulin, as mentioned above. 
STZ does have a well-known pathway leading ultimately to cytotoxicity to $\beta$ cells. It is known that STZ is a nitrous oxide (NO) donor, which has been observed to be destructive to pancreatic islet cells, in particular to their DNA. The mechanism of action is that when $\beta$ cells metabolize NO, their DNA undergoes fragmentation [5]. When exposed to STZ, $\beta$ cells exhibit telltale signs of NO poisoning: increased activity of guanylyl cyclase as well as amplified formation of cGMP. It is the cytotoxicity to these vital cells that initiates apoptosis, or cell death, thus rendering the rodent unable to take up any glucose from the bloodstream [5]. This excessive glucose now collecting in the bloodstream continues to increase in concentration with time. Upon injection, the pancreatic $\beta$ cells take up the STZ via the glucose transporter GLUT2. Once it is taken up by these cells, STZ inhibits oxygen consumption in the cell's mitochondria. Now in a hypoxic state, these mitochondria are then left unable to produce ATP at the same rate as before and the cells die as a result [5]. Over time, more and more $\beta$ cells die, resulting in a complete inability to take up glucose from the bloodstream. The major effect of this is excess blood sugar, or hyperglycemia. The use of STZ as a mechanism to induce insulin-dependent diabetes is an accepted method due to the wide body of literature surrounding its biochemical action as well as time-tested results. 


\section{Chapter 7}

\section{Materials \& Laboratory Fixtures}

The final product of this research is a fully-developed hardware setup, software application, and surgical protocol to obtain optimum neuron response to sciatic nerve stimulation. Prior to the specifying the steps involved in performing the surgery, it is important first to identify the required materials and supplies. Beginning from the first stage of the experiment all the way to the last, they are listed below in Table (2).

Table 2: List of Instruments

\begin{tabular}{|c|c|}
\hline Surgical Tape & Large Surgical Scissors \\
\hline Forceps & Inox \#7 Forceps \\
\hline Nose Cone Clamp (the stand) & Gauze Pads (2" x 2") (5) \\
\hline Saline Solution & Small Surgical Scissors \\
\hline Surgical drape & Cotton Swabs \\
\hline Electric Hair Clippers & Laboratory Gloves \\
\hline Protective Bite-Proof Gloves & Syringe \\
\hline Needles & Sharps Container \\
\hline Depilatory Cream & Rat-specific Nose Cone \\
\hline
\end{tabular}

\section{Surgical Tape:}

This roll of tape is meant to be used to fix the hindlimb of the anesthetized rodent to the hair clippers as shown below. This elevates the leg and allows for easier access to the sciatic nerve upon exposure. 


\section{Small Surgical Scissors:}

The narrow tips of these extremely sharp instruments avoid the potentially clumsiness associated with scissors, especially when making fine cuts in anatomically deep areas. The precision afforded by these instruments help avoid inadvertent bleeding because of an accidental cut.

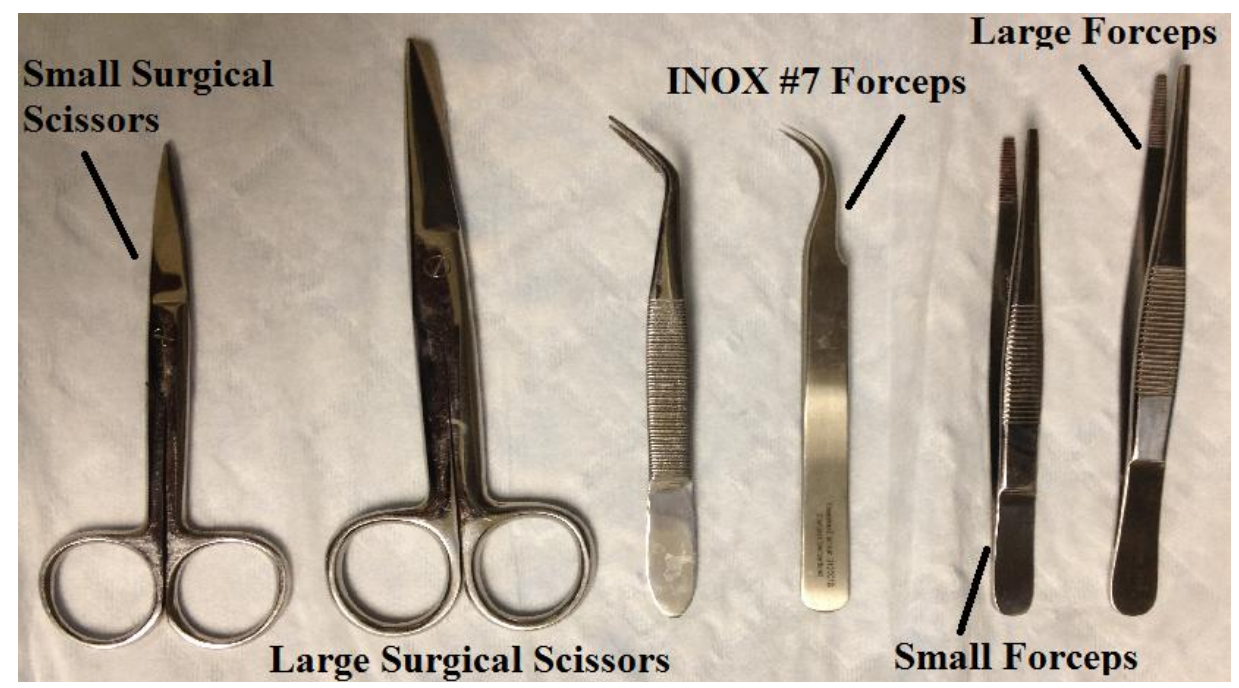

Figure 8: Layout from left-to-right: small surgical scissors, large surgical scissors, curved forceps, Inox \#7 forceps, small forceps, large forceps.

\section{$\underline{\text { Small Forceps: }}$}

These small forceps are used to delicately grip onto soft tissue during the surgery. They allow for fine control and manipulation of tissues so as to inflict as little trauma as possible for tissues not meant to be dissected.

\section{Large Surgical Scissors:}

These can be used to make the initial incisions into the skin of the animal. Depending on the size of the area to be incised into, however, a scalpel or small surgical scissors may be used as well. Again, the choice is up to the discretion of the surgeon. 


\section{Inox \#7 Forceps:}

These specialty forceps feature curved pointed tips, which allow for more comfort in the wrist during use as well as extremely precise control over placement. Their characteristically narrow tips also make for an ideal instrument for blunt dissection.

\section{Saline Solution:}

This solution is used primarily as a flushing agent to prevent nerve desiccation. Putting it inside a $3 \mathrm{ml}$ syringe with a needle assists with greater control over where the saline is flushed to. It is also conductive, and is to be flushed through the electrode immediately before setting it around the nerve. This will be explained in greater detail later.

\section{Surgical Drape:}

This surface cover for the table helps with absorbing any fluid excess that flows off the animal during surgery.

\section{Electric Hair Clippers:}

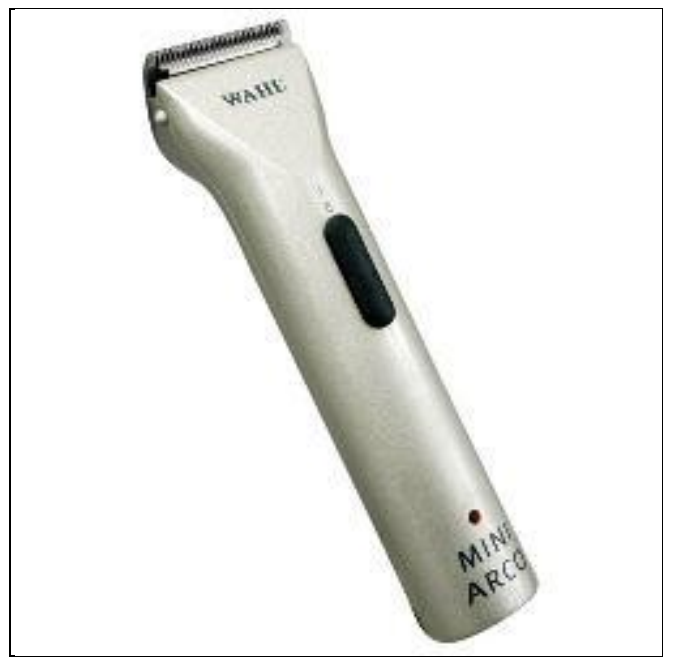

Figure 9: Electric hair clippers used to remove the majority of fur before surgery. 
Due to the high density of the fur on the animal, it is recommended that these be used to remove most of the fur before depilatory cream is used. This greatly reduces surgery time and offers the surgeon a hair-free hindlimb for a neater surgery.

\section{Protective Bite-Proof Gloves:}

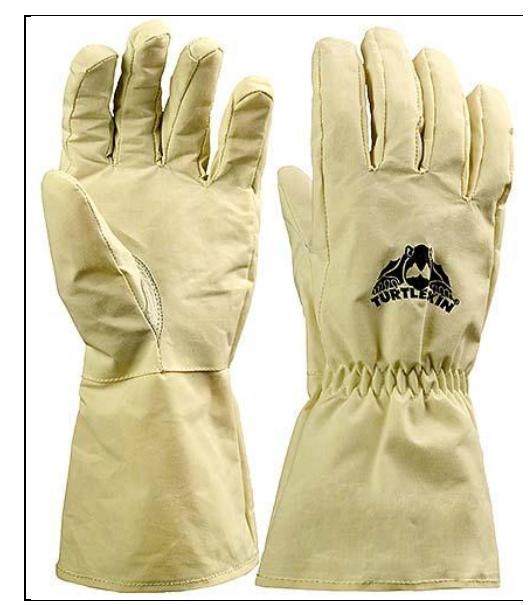

Figure 10: bite-proof gloves are meant to protect the handler in case of a bite.

The use of these is recommended for animals which may be prone to biting during handling. Though their use is not required, it allows the animal handler a degree of comfort that often results in more relaxed technique for rodent handling. Ultimately, their use is up to the discretion of the handler.

Syringe \& Needle:

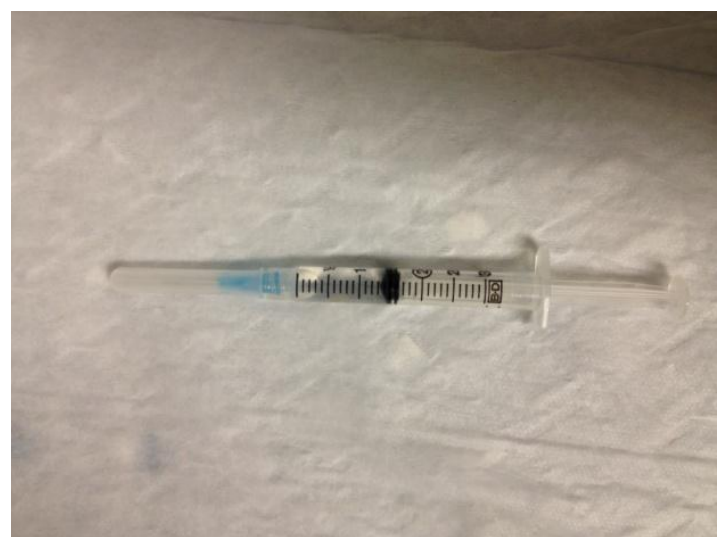

Figure 11: Syringe and needle used to apply saline solution during procedure. 
These are highly recommended for precise control over the flow of saline solution. They are used to deposit saline solution where it is required during surgery.

\section{Depilatory Cream:}

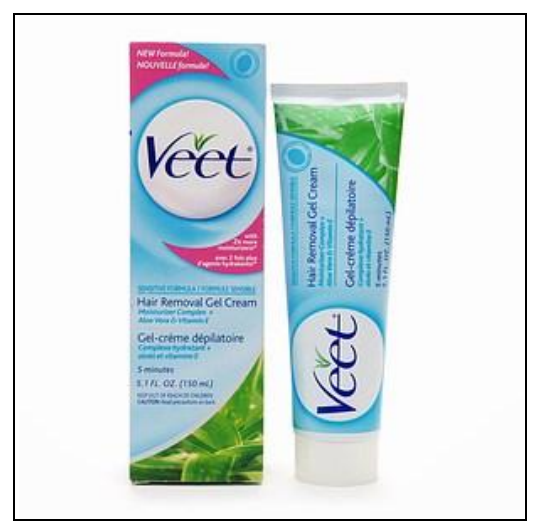

Figure 12: Depilatory cream removes any remaining fur before surgery.

Using gloved hands, the cream is deposited on areas of the hindlimb which have already been trimmed for excessive hair. Using a cotton swab or a small gauze pad, spread the cream on the surface and leave it on for at least 2 minutes before gently wiping off with a gauze pad to expose the skin.

\section{Cotton Swabs:}

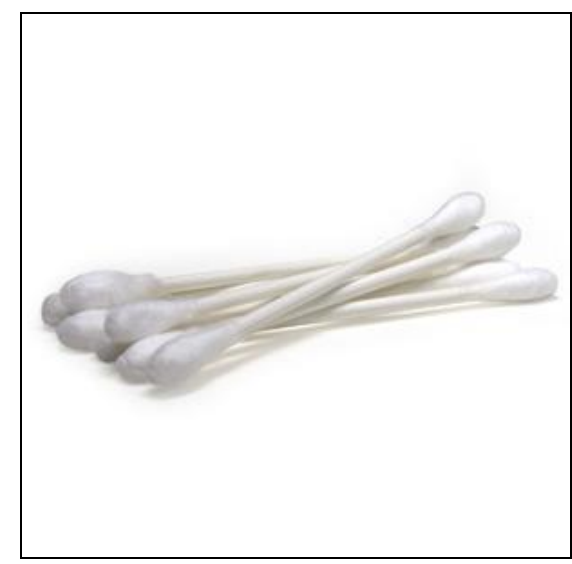

Figure 13: Cotton swabs may be helpful in stopping bleeding.

These can be used to apply depilatory cream to the leg before the first incision is made. Also, if an artery or vein is accidently cut during surgery and bleeding occurs, the head of a cotton swab may be placed over the wound to help stop bleeding. If it does not interfere with the 
remainder of the procedure, surgery may continue while it is sitting there. However if it obstructs the surgeon's field of view, it is recommended that a few minutes be taken to allow the blood to clot before continuing.

Gauze Pads (2" x 2"):

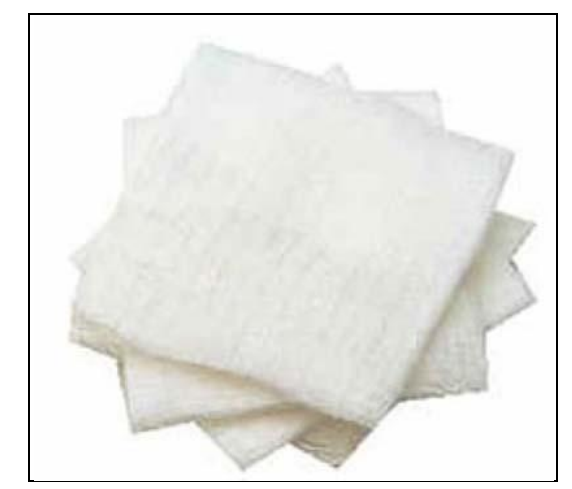

Figure 14: Gauze pads are a widely used tool during surgical procedures.

Several of these are recommended to be on hand during any procedure. Their broad applicability to any procedure makes them an invaluable asset. They help to clear the open surgery area immediately after saline flushing as well as collecting any fluids which may stray from the specimen.

\section{Laboratory Gloves:}

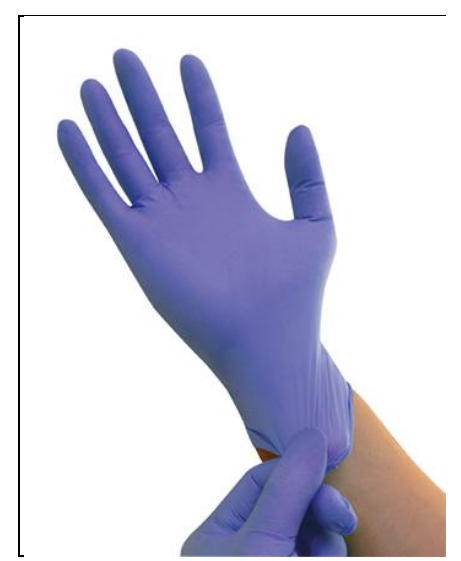

Figure 15: Lab gloves are required to protect the surgeon from particle transmission when handling animals. 
These are required for all procedures when handling rodents. They also help to maintain a sanitary field and laboratory. Their use is recommended from surgery table setup all the way to instrument washing and for everything in between.

\section{$\underline{\text { Sharps Container: }}$}

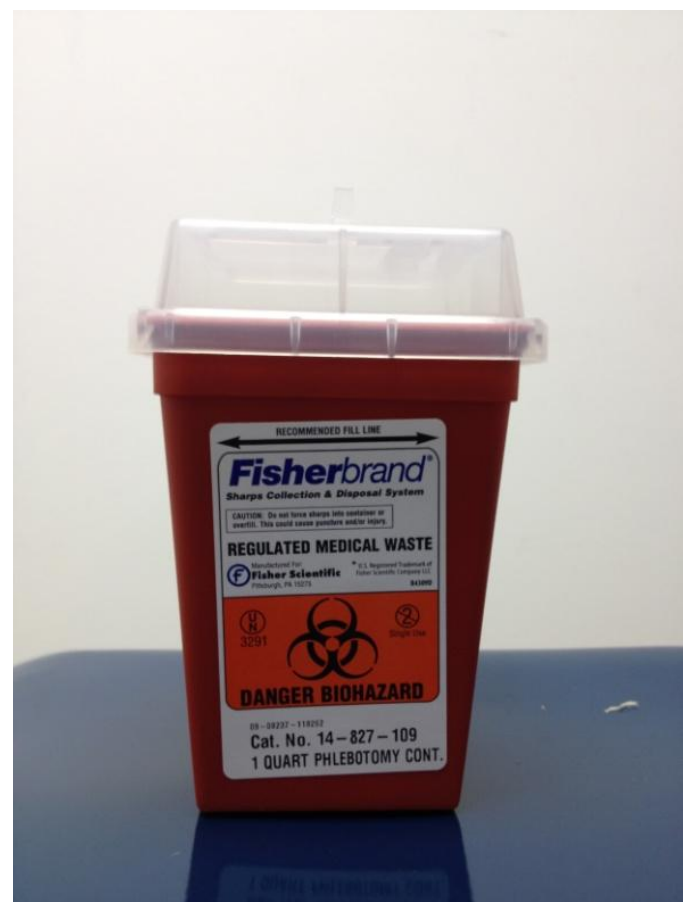

Figure 16: The Sharps container contains discarded needles and syringes.

This is the only appropriate location to discard sharps such as needles and syringes. This bin is identified by its red color and its sharps labeling.

\section{$\underline{\text { Rat-Specific Nose Cone: }}$}

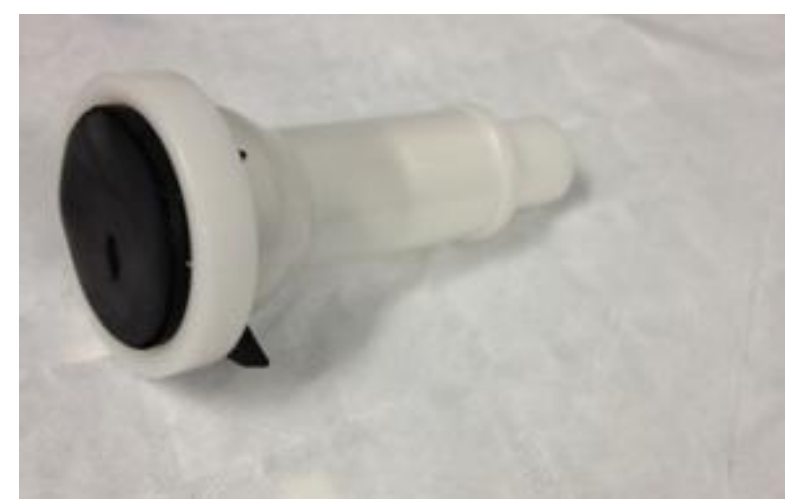

Figure 17: The nose cone fits over the rat's nose to deliver anesthetic. 
This is a specialty nose cone covered by a diaphragm. The diaphragm is the sheet of synthetic material pulled taught over the large end with the hold cutout for the rat's nose. Note that the cutout for the rat is larger in diameter than that for a smaller rodent such as a mouse. The nose cone connects directly to the anesthesia machine and is the part that directly interfaces with the animal being anesthetized.

The table below outlines the fixtures in the laboratory required for performing this procedure. Each of the items in the table is considered a fixture of the lab because none of them can be removed, unlike supplies which can be transported from room-to-room.

Table 3: Laboratory Fixtures

\begin{tabular}{|l|l|}
\hline Faraday Cage & Anesthesia Cart \\
\hline Isoflurane Anesthetic & Oscilloscopes (2) \\
\hline Computer & Electrode Cuff \\
\hline Differential Amplifiers (2) & Voltage-Controlled Current Source \\
\hline Induction Chamber (in surgical suite) & Small Oxygen Tanks (2) \\
\hline Recording Site Electrode Adapters (2) & Electrode Adapter Boxs (2) \\
\hline
\end{tabular}

\section{Faraday Cage:}

This is the location of all electrophysiology procedures. The electrical insulation of the cage assists with performing electrical measurements with a higher signal-to-noise ratio than would be possible outside of the cage. It is also the location of the differential amplifiers and the voltage-controlled current source.

\section{Isoflurane Anesthetic:}

This is the inhalant used to anesthetize the rodent. It is a highly volatile liquid anesthetic, so exposure to air results very quickly in evaporation. Thus such exposure time must be reduced 
so as to minimize the amount lost to evaporation. All use must be documented, as it is a controlled substance.

\section{Computer:}

This is the platform used to activate and control the stimulation and recording software.

\section{DAM50 Differential Amplifiers:}

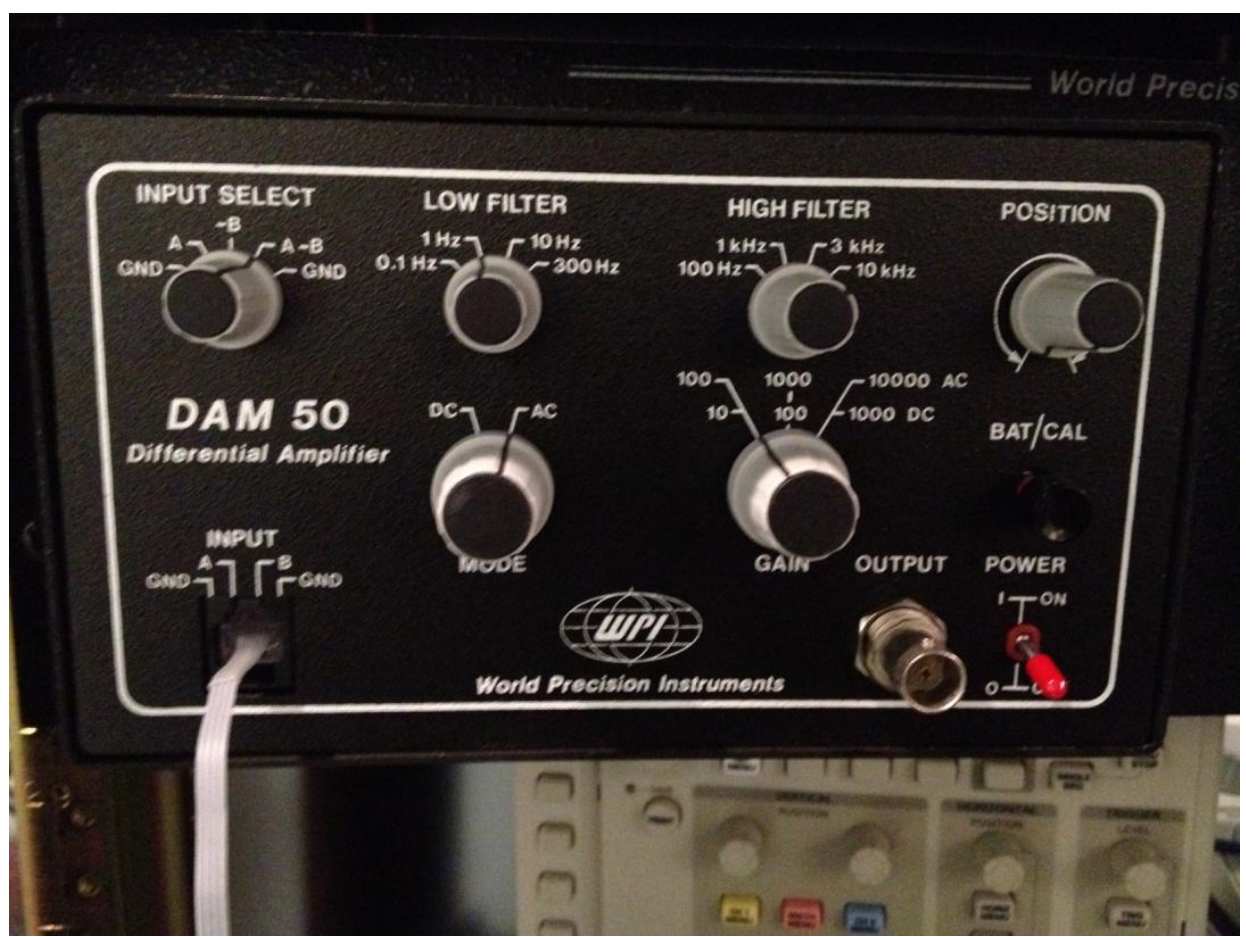

Figure 18: Differential Amplifier used for signal acquisition and noise filtration.

These are two differential amplifiers used to acquire the signals and filter out the noise associated with electrophysiology recordings. 


\section{Induction Chamber:}

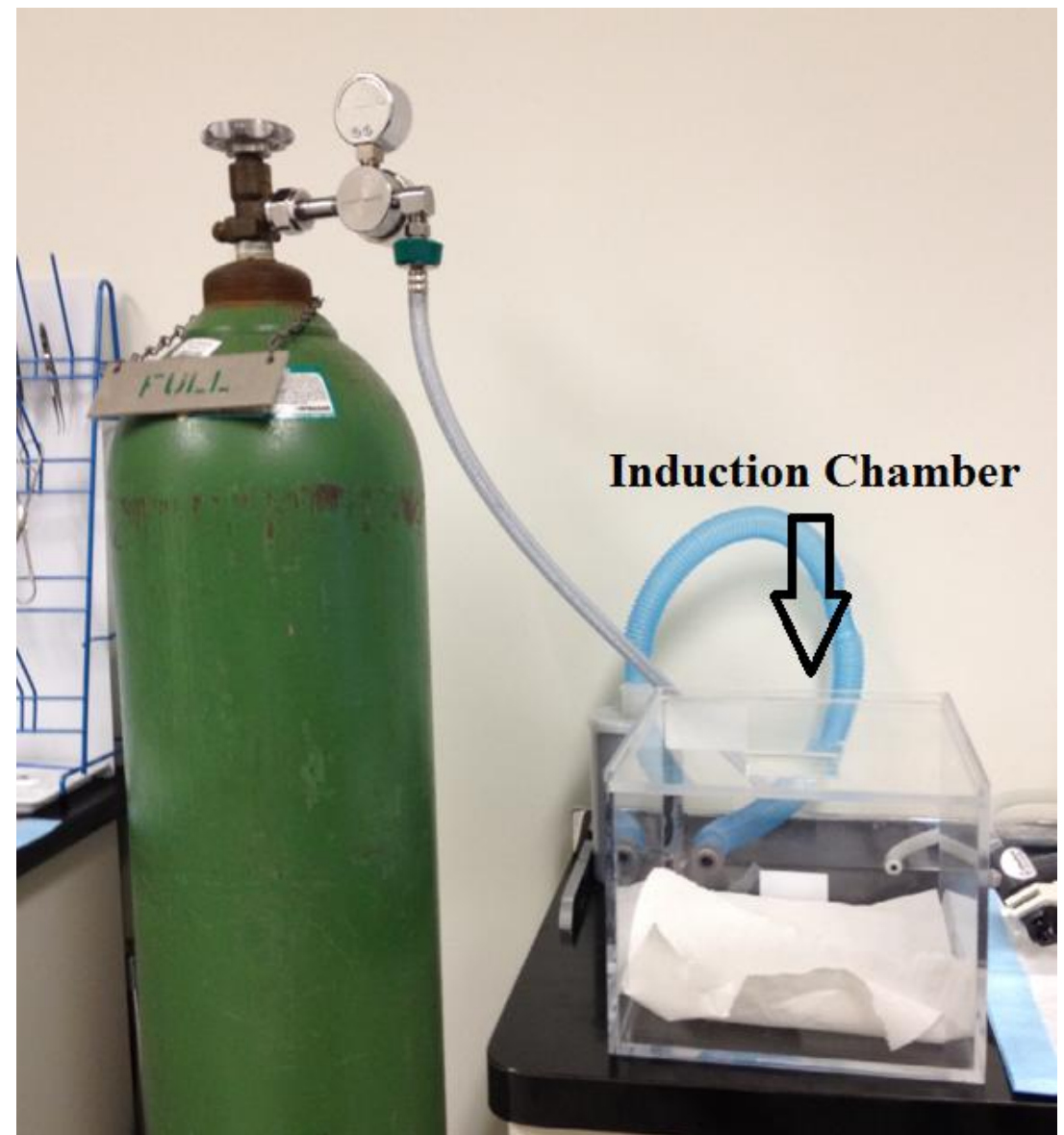

Figure 19: The induction chamber is the first stop for the rodent being anesthetized.

This is the plastic container into which the rat is placed before surgery in order to induce initial anesthesia. Once the rodent is fully anesthetized, it is to be left inside the chamber before quickly carrying it into the Faraday Cage. 


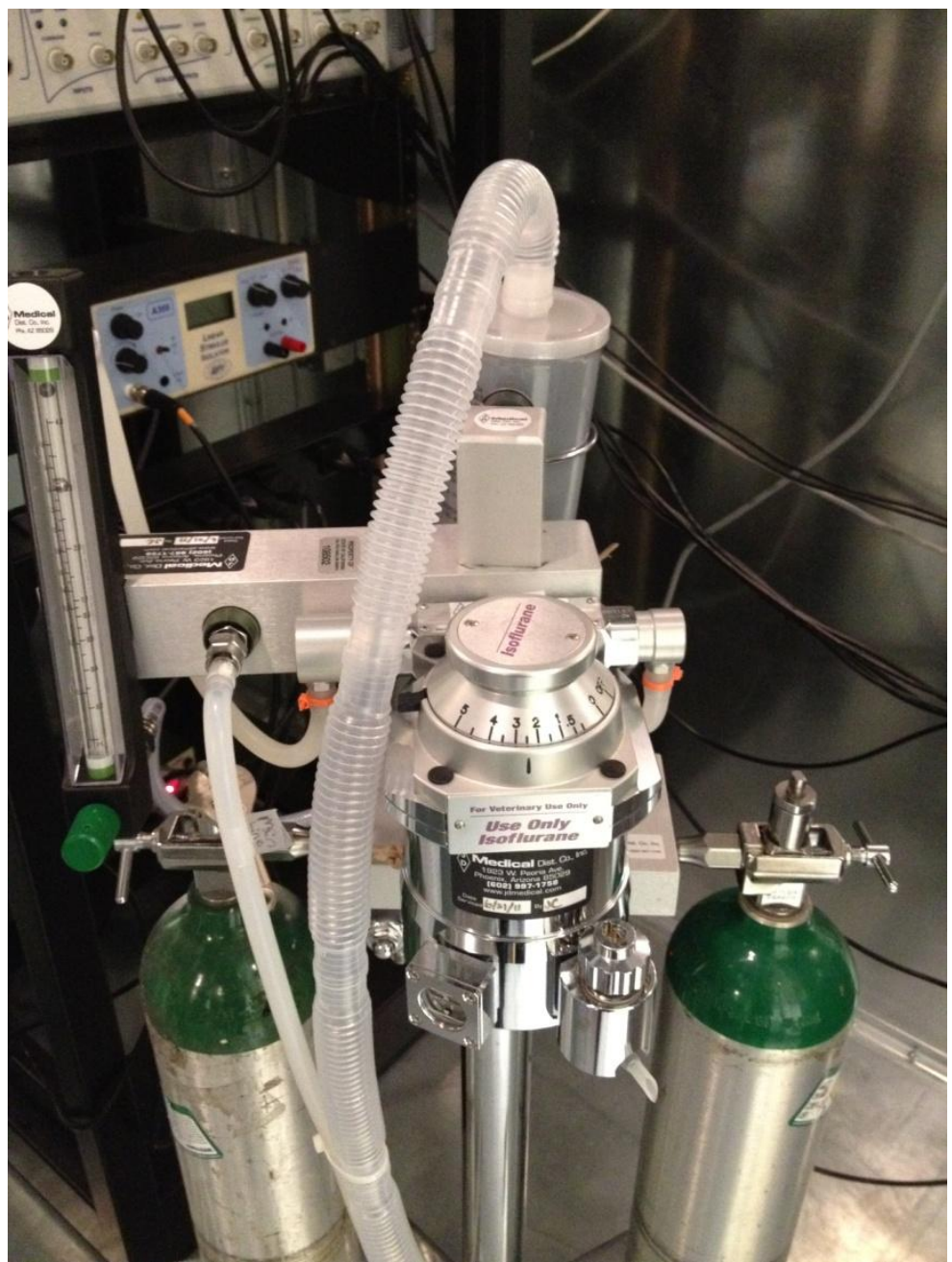

Figure 20: The anesthetic cart delivers anesthesia and oxygen to the subject.

This is the device which is used to control oxygen flow and anesthesia infusion percentage. Used both for the induction chamber and the Faraday Cage, full oxygen tanks are absolutely imperative for surgery. 


\section{Oscilloscopes:}

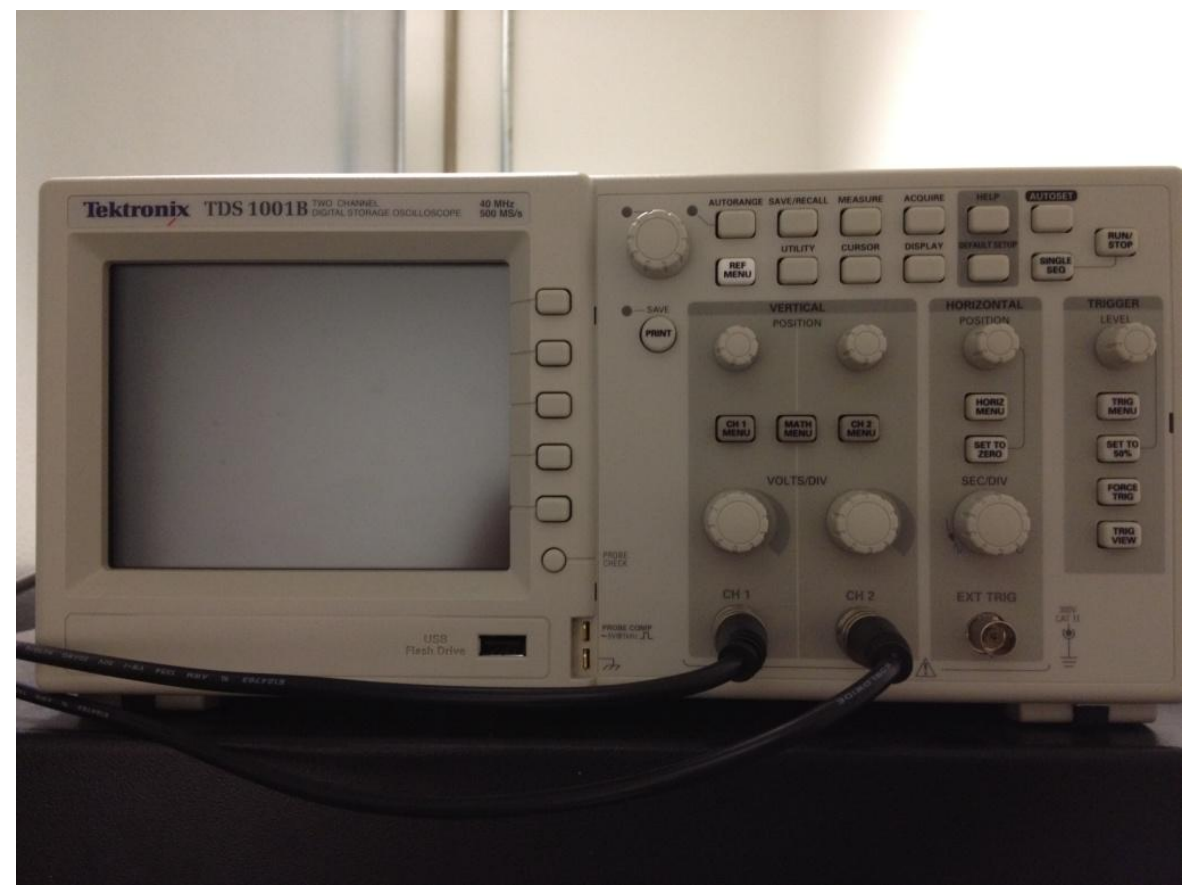

Figure 21: The oscilloscope is used to visualize the waveforms associated with the stimulus and the response.

These devices allow both the surgeon and the computer operator to visualize the stimulation waveform and the response waveform. The volts/div knobs control the potential scale that is seen on the display.

\section{Electrode Cuff:}

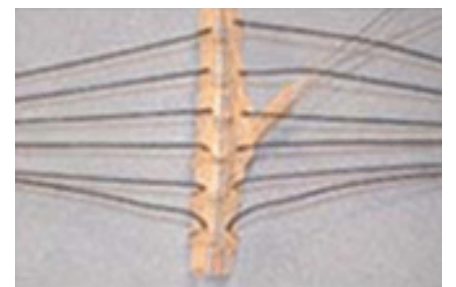

Figure 22: The electrode cuff fits directly over the nerve.

This is the specialty cuff which is connected to the current source. It transmits the stimulation signal from the current source directly to the nerve. Before application to the nerve, the inner surface is to be flushed with saline in order to promote conduction between the nerve- 
electrode interface. Each lead is marked by a colored bead. The distance between consecutive leads (and therefore between their corresponding cuffs) is $1 \mathrm{~mm}$.

\section{Voltage-Controlled Current Source:}

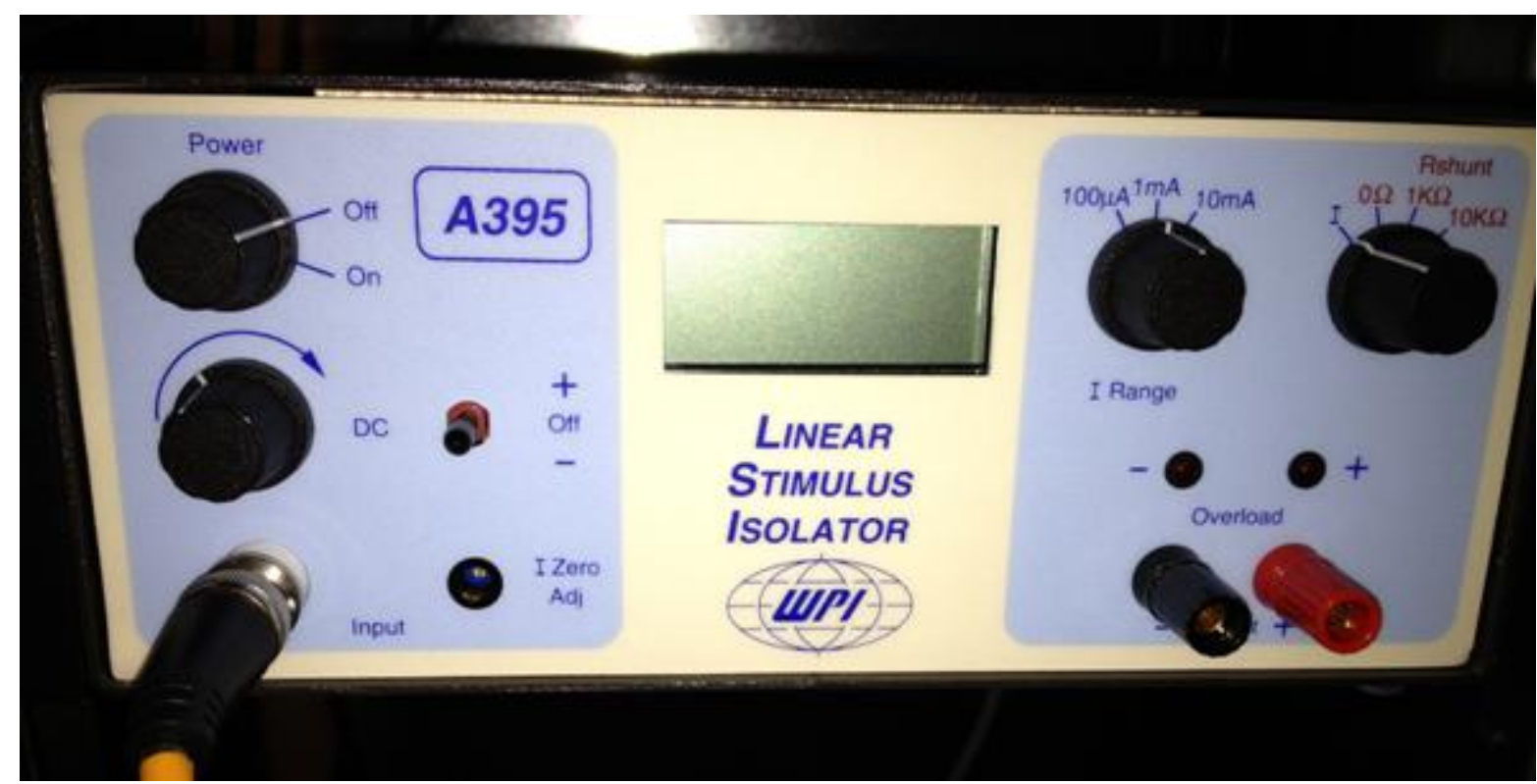

Figure 23: The voltage-controlled current source delivers the pulses to the cuff.

This is the stimulator used to deliver current to the nerve. The current is applied as a square pulse train directly to the nerve.

\section{Small Oxygen Tanks:}

There are two green oxygen tanks connected to the anesthesia cart. Once a surgeon becomes proficient at performing the procedure efficiently and quickly with minimal delays, as little as one tank of $2000 \mathrm{psi}$ should suffice for the entire procedure from beginning to end. But having two full tanks allows a larger time buffer to account for unforeseen delays during the procedure. 


\section{$\underline{\text { Recording Site Electrode Adapters: }}$}

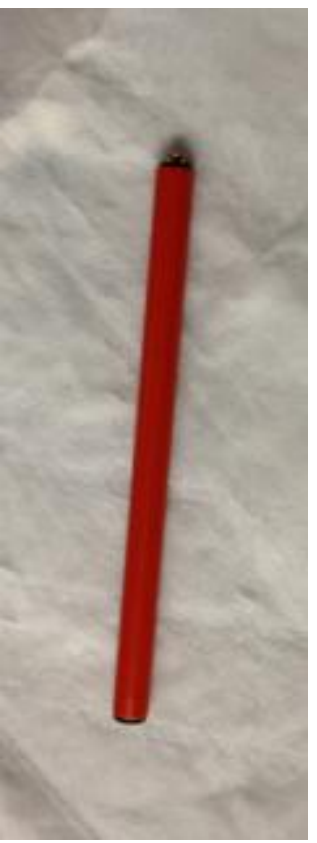

Figure 24: Electrode adapters connect the recording site leads to the rest of the apparatus.

These slender red rods are adapters that allow the electrode cuff leads from the recording sites to connect to the electrode adapter boxes.

\section{Electrode Adapter Box:}

This is the apparatus that creates a common ground between the recording sites, and that connects the recording sites to their respective DAM50 differential amplifiers. They are each wired according to a specific diagram, shown below.

\section{Student Software:}

\begin{tabular}{|l|l}
\hline LabVIEW stimulation software & MATLAB signal analysis software
\end{tabular}

This software is available directly on the computer in the lab. 


\section{LabVIEW Stimulation Software:}

This student-written software outlined below controls the stimulation and signal recording protocol.

\section{MATLAB Signal Analysis Software:}

This post-processing software receives the text files assembled by LabVIEW and displays them as a graph, along with key statistical information. 


\section{Chapter 8}

\section{Methods}

To obtain acceptable action potential recordings requires the work and cooperation of two people during the surgery. The surgeon directly handles the animal and sets the hardware, while the operator prepares the software and initiates the stimulation and recording software. The heavily involved nature of this procedure requires that the surgeon and the operator prepare the surgical instruments, laboratory equipment and prepare the animal for surgery in an efficient and timely manner. The steps outlined below ensure that all these points are met and that the procedure can be carried out with minimal delay. In the interest of tractability, the surgeon's responsibilities are divided into 4 main routines represented by the acronym PAST.

PAST: Preparation, Access, Stimulation, and Termination.

Each step in the PAST method is necessary for obtaining successful stimulation and recordings of nerve response to a pulse. The motivation behind this method is not to simply obtain the results, but rather to do it in a timely manner. Once the nerve is exposed to air, it begins to dry out and must be kept in a moist environment. Also, making too large an incision increases the likelihood that more nerve than necessary is isolated which reduces the viability time of the nerve. Following the steps outlined by this method ensures that the nerve is isolated only minimally and for a very short duration so that any damage done by exposure is minimized. 


\section{1: Preparation}

This routine serves to organize all the instruments and equipment before surgery is begun. A vital part of this is to ensure that all materials are functional and accounted for. This includes the anesthesia cart, oxygen tanks, isoflurane, surgical instruments, and electrode cuff. It is recommended that the surgeon use the checklist below to account for these materials. During this time, the operator may opt to prepare the software on the computer.

\section{Materials Checklist}

\begin{tabular}{|c|c|c|c|c|c|}
\hline 0 & Surgical Tape & ○ & Forceps & ○ & Nose Cone Clamp \\
\hline O & Saline Solution & O & Surgical Drape & ○ & $\begin{array}{l}\text { Electric Hair } \\
\text { Clippers }\end{array}$ \\
\hline O & Bite-Proof Gloves & o & Needles & O & Depilatory Cream \\
\hline 0 & $\begin{array}{l}\text { Large Surgical } \\
\text { Scissors }\end{array}$ & O & Cotton Swabs (5) & O & Lab Gloves \\
\hline O & $\begin{array}{l}3 \mathrm{ml} \text { Syringe \& } \\
\text { Needle }\end{array}$ & O & Sharps Container & 0 & $\begin{array}{l}\text { Rat-specific Nose } \\
\text { Cone }\end{array}$ \\
\hline 0 & Isoflurane Bottle & O & Anesthesia Cart & O & Induction Chamber \\
\hline 0 & $\begin{array}{l}\text { Full Oxygen Tanks } \\
\text { (2) }\end{array}$ & O & Electrode Cuff & & \\
\hline
\end{tabular}

It is at this point that the rat should be sitting in its carrier cage with bedding, food, and water available. While pre-surgery preparation continues, place the carrier in a quiet corner of the Faraday Cage with a cover over the top to limit exposure to light. This helps keep the animal unstressed.

1. Put on a pair of lab gloves. 
2. Follow the opaque white hose from the induction chamber all the way to the anesthesia cart, ensuring that it is connected. The line is labeled "IND" on the end that connects to the anesthesia cart.

3. Check the isoflurane level through the viewing window of the anesthetic cart. Unscrew the cap above it and fill it with isoflurane until the level approaches the upper white line. Figure (24) below shows a low anesthetic level seen at the bottom of the indicator.

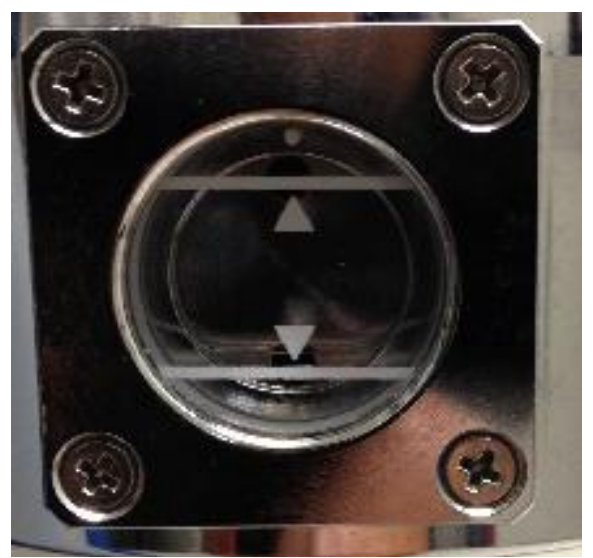

Figure 25: The level indicator on the anesthesia cart.

4. Check the pressure of the large green oxygen tank adjacent to the induction chamber to ensure that it is out of the red "Refill" zone.

a. Open the screw cap on top of the tank and look at the pressure gage. The needle should be out of the red "Refill" zone.

b. Close the tank.

5. Test the pressure level of the small oxygen tanks:

a. Refer to the back end of the anesthesia cart. There are two threaded connectors, one horizontal and the other beneath it vertical. First unscrew the green one from the horizontal one and replace it with the silver hex-shaped one from the vertical one. 


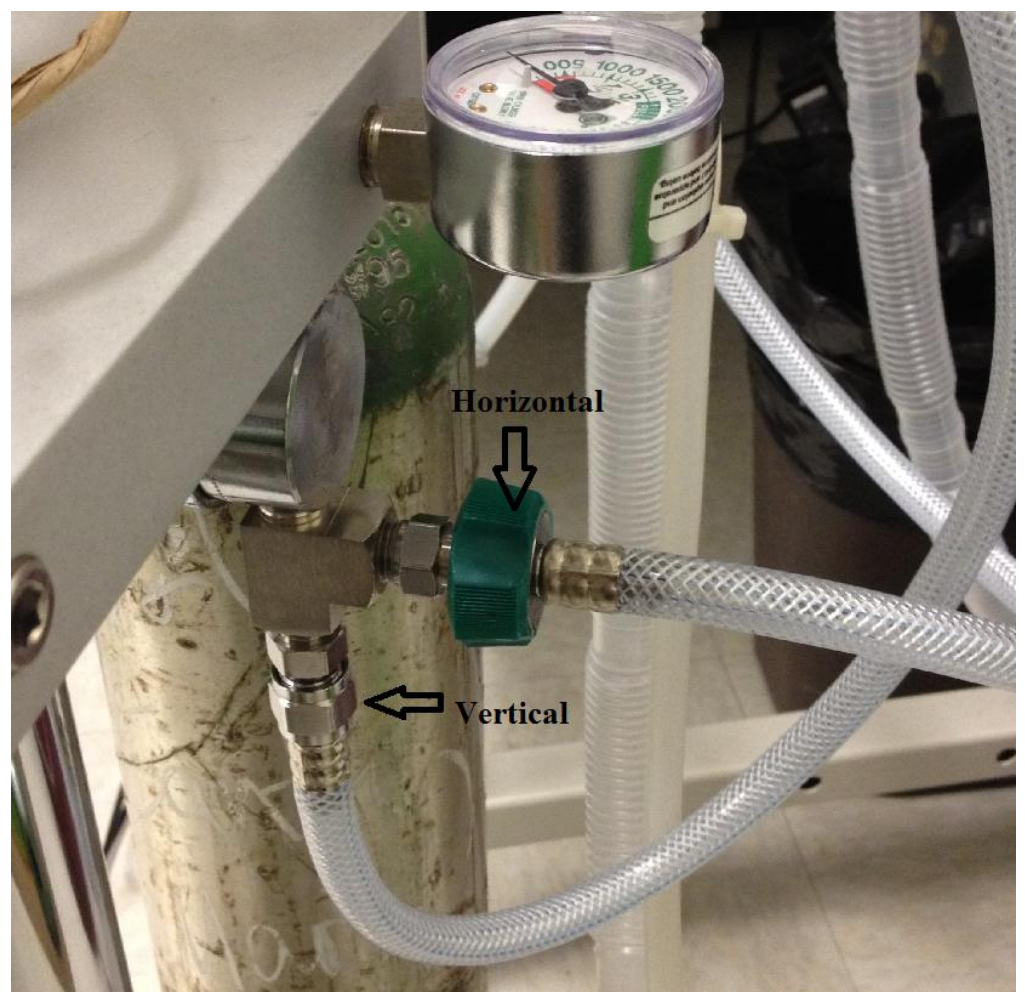

Figure 26: The horizontal and vertical threaded connectors on the anesthesia cart.

b. With the crescent wrench, open one of the oxygen tanks, ensuring that the green flow rate valve is closed. If it is closed, the ball will be resting at the bottom of the tube.

c. While it is open, look to the cart's pressure gage. A full tank will read 2000 psi.

d. Close the oxygen tank with the wrench.

e. Slightly unscrew the hose from the horizontal threaded portion briefly to release the air trapped in the hose. A hissing sound should be heard..

f. Retighten the hose with the wrench.

g. Now use the wrench to open the second tank in the same manner. Again, it should read 2000 psi. 
h. Close the oxygen tank, unscrew the line and replace it on the vertical threading.

i. Reattach the green collar to the horizontal threaded section.

6. Ensure that the surgery table in the Faraday Cage is close enough in proximity to the tall shelving unit with the oscilloscope, differential amplifiers, and current source.

7. Place a surgery drape over the table.

8. Arrange the surgical instruments on the silver microscope table adjacent to the surgery table:
a. Large Surgical Scissors
b. Small Surgical Scissors
c. Forceps
d. Inox \#7 Forceps
e. Surgical Tape
f. Electrode Cuff

9. Carefully screw the syringe into a needle and fill it with saline from the saline bottle. Place it alongside the rest of the instruments.

10. Place the gauze pads near the instruments.

11. On the shelving unit supporting the current source, flip the red switch to the "ON" position. This charges the source in preparation for stimulation.

12. Place the nose cone clamp on the corner of the table. This will be used to stabilize the nose cone in place during surgery.

13. Don the bite-proof gloves.

14. Slide the lid off of the induction chamber. 
15. Carefully restrain the rat and place it gently into the induction chamber, closing the lid quickly but calmly after it.

16. Place the rat carrier and the bite-proof gloves away.

17. Open the large green oxygen tank until the needle rises. This causes oxygen to flow into the induction chamber.

18. While depressing the black tab on the anesthesia cart, turn the large silver control knob until the black tick mark aligns with $2.5 \%$.

19. At this point, the anesthetic is being delivered to the rat. One will observe slowed movement, and collapse.

20. Check the rat for proper anesthetic plane.

a. Carefully tilt the induction chamber so that the rat has an opportunity to roll over and subsequently right itself. If it resists rolling over, then not enough anesthetic has been delivered, in which case the surgeon will wait another 2 minutes before repeating the tilt test. If it fails a second time, increase the infusion rate from $2.5 \%$ to $3 \%$ and wait another 2 minutes.

b. Once the rat has lost the ability to right itself in the tilting induction chamber, it is prepared for surgery.

c. Turn off the large oxygen tank and drop the infusion rate to $0 \%$. Do not open the lid to the induction chamber. Do this last because once the rat leaves the chamber, which has residual anesthetic it will begin to awaken without a constant source of anesthetic inhalant.

21. Disconnect the anesthetic hose from the anesthesia cart. This frees it from place and allows it to roll to other locations. 
22. Carefully roll it from the surgery suite to the Faraday Cage.

23. Connect the existing anesthetic hose from the cart to the port where the old anesthetic hose used to be.

24. Remove the clear plastic nose cone holder from the cart and replace it with the ratspecific one.

25. Use the wrench to open one of the tanks, but ensure that there is still no flow. Again, the needle will rise.

26. Place the nose cone in the nose cone holder on the surgery table so that it is low enough that the rat's nose is at the same height.

\section{2: Access}

1. Open the induction chamber and carry the rat to the surgery table where the anesthesia cart is set up.

2. Quickly place the rat on its back with its nose resting near the nose cone.

3. Once placed there, turn the green flow rate knob up so that the silver ball floats up to the 3 LPM (liters per minute) tick mark.

4. Turn the isoflurane infusion rate up to $2.5 \%$.

5. Allow the rat to rest under the current oxygen flow rate and infusion rate for a few minutes.

6. Now it is appropriate to take a ventilatory rate reading from the animal:

a. Set a timer to beep after 30 seconds have elapsed.

b. When the timer begins counting down, count how many breaths the rat takes before the beep.

c. Multiply that number by 2 to give the breaths per minute. 
7. To verify that the rat is under proper anesthesia, use forceps to pinch one of the toes on the hindlimb. If the rat retracts its leg, this is evidence that it is not receiving enough anesthetic. To remedy this, slightly increase the anesthetic concentration from $2.5 \%$ to $3 \%$. Changes to the anesthetic concentration should be made by increments of half a percent in order to safeguard against over anesthetizing the animal.

8. Wait another minute before repeating the pinch test. When the pinch test results in no retraction, proper anesthesia has been achieved and the procedure can begin.

9. Using a gentle motion, use the electric clippers to trim the hair surrounding the hindlimb where the incision is to be made. The appropriate area extends from the proximal thigh to the heel, removing all hair from the medial aspect of the hindlimb. Sweeps should be made against the grain of the hair applying very little pressure against the skin.

10. Once finished, apply a small amount of depilatory cream to a piece of gauze. This will be rubbed onto the region where hair has been trimmed. Allow this to sit for 2 minutes.

11. With another piece of gauze, wipe off the depilatory cream and the remaining hair along with it. There should be a smooth exposed area of skin remaining. Discard the used gauze pads.

12. Now with the electric hair clippers turned off, place them beneath the rodent's right hindlimb. It may be helpful to tear off a piece of surgical tape about $1 \frac{1 / 2}{2}$ to 2 " long and use it to tape the rodent's right hindlimb onto the clippers. This step helps to simultaneously stabilize the leg and expose the sciatic nerve. The angle at which the leg is now placed is also more ergonomically desirable. Refer to the photo below 
13. The first incision should be made from the origin of the proximal hindlimb distally to the heel. Using the forceps in one hand and the small surgical scissors in the other; incise gently through the outermost layer of skin in an arc shape, following the slight curve of the leg.

a. If bleeding occurs during any part of the surgery resulting in an obstructed field of view, simply cut the end off of a cotton swab and gently place it over the area that is bleeding. Wait a few minutes for the wound to clot. This will help the area to clot sooner.

14. Once the incision is complete, the femoral artery and vein should be visible embedded in the gracilis muscle. These landmarks indicate that the section of the sciatic nerve that is optimal for electrode placement is just medial and inferior to these by $1 / 2$ " in each direction. Exposing any section of nerve distal to this section will result in poor electrode placement due to the obstruction of other nerves stemming from the sciatic. A good rule of thumb is to aim for the sciatic nerve that is proximal to the patella. That way, the entire lower hindlimb is left undisrupted.

15. The following muscle dissections are to be made in an orientation perpendicular to the axis of each muscle. Dissecting along the axis of a muscle is inefficient and requires more cuts, which increases undue trauma to the area. 


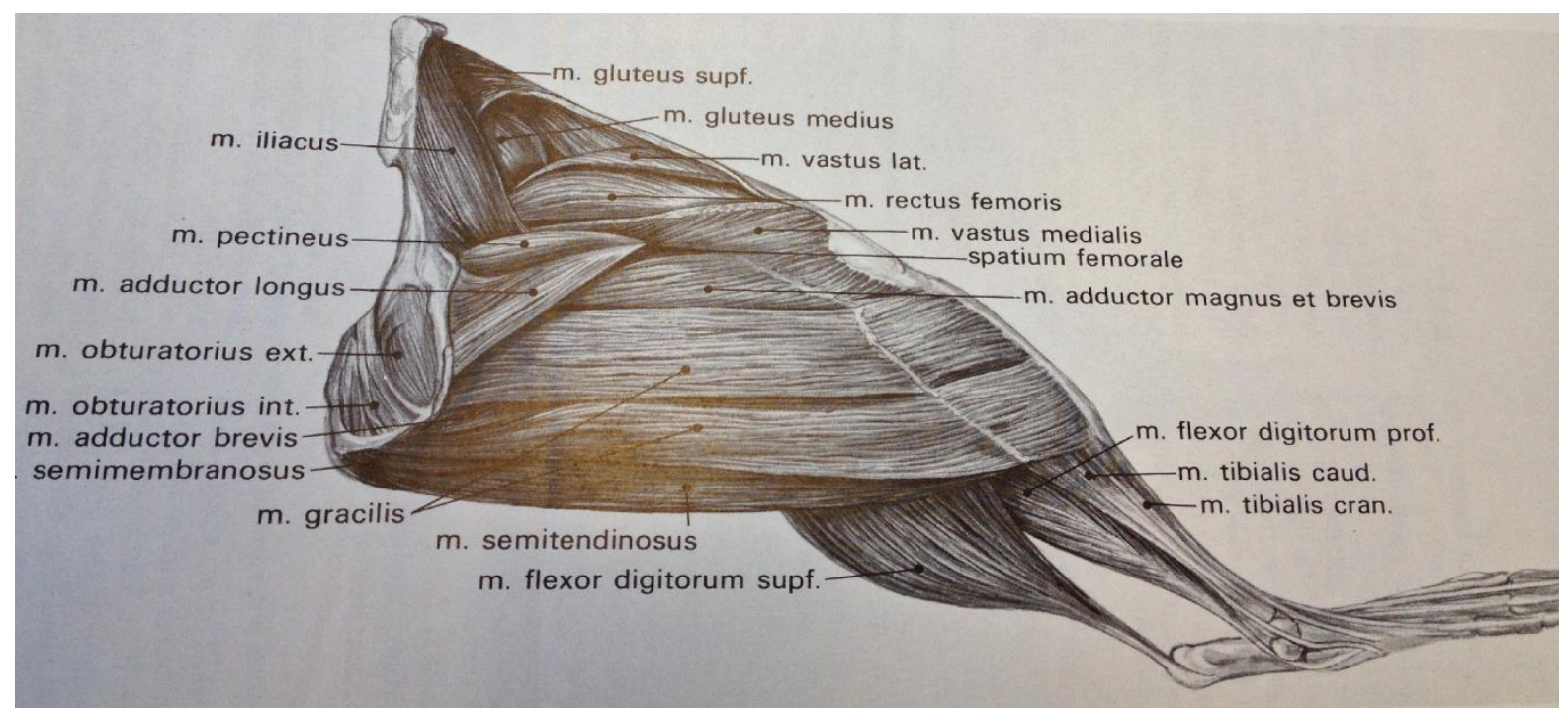

Figure 27: Rat leg muscle anatomy [6]

16. Begin by carefully dissecting the gracilis cranialis muscle inferior to the femoral artery. This relatively large muscle can be identified by its wide shape overlaying all deeper muscles. Once dissected, reflect it out of the way.

17. Just inferior to the gracilis lays the gracilis caudalis. This muscle must also be dissected in the same manner as the gracilis cranialis in order to free the movement of the leg when applying the electrode.

18. Now it is time to transect the semitendinosus, which is the most inferior muscle that will be transected in this procedure. When transecting this muscle, take extra care not to inflict any trauma on any surrounding tissues because this lays in very close proximity to the sciatic nerve, which must remain undamaged.

19. Now, a branch of the sciatic nerve should be visible. If not, dissect the adductor brevis and adductor longus to give the leg some more flexibility. The sciatic nerve can be identified by its thick, white, rope-like morphology.

20. With Inox \#7 forceps, carefully blunt dissect around the sciatic nerve to expose and isolate a length of about $8 \mathrm{~mm}$ so that the entire cuff can fit into the opening. 
21. Once completely isolated from surrounding tissue, the sciatic nerve is ready for electrode placement.

22. Use the syringe to apply a small amount of saline onto the inside portion of the electrode. This promotes complete conductivity between the nerve and electrode.

23. The two ends of the electrode have knots protruding from each end of each cuff. They are tied together in two bundles for ease of manipulation. Using Inox \#7 forceps slid beneath the nerve inverted (tip pointed up), place one knot into their grasp and pull the cuff below the nerve and around it.

24. Using gentle motion, ensure that the nerve is resting gently inside the cuff without strain in any direction. Physical strain on the nerve can cause action potentials, which would corrupt the data recordings.

\section{3: Stimulation}

The protocol for optimum stimulation involves integration of the software program with the electronic hardware available. The user interface on the LabVIEW stimulation software prompts the user to enter the following input parameters. These are outlined below along with the justification for each parameter in Table (3) below. This is a twostage process that involves an initial low-resolution stimulation and reading of the nerve in order to ascertain the minimum voltage required to cause an action potential. That is, the first stage stimulation only serves to give the operator a ballpark figure of the initial stimulation voltage, so small current steps are unnecessary). Following this stage is the actual high-resolution stimulation stage that gives the in-depth information about the nerve. The advantage of determining the initial stimulation voltage is that a high resolution-smaller current step — can be afforded. Referring back to Chapter 4, the 
group delay method is premised on the assumption that the current step is small enough to invoke the addition of only a single nerve fiber at a time. Thus, the smallest current step possible is advantageous in the sense that it is more likely that a single additional fiber will be stimulated with each voltage increase.

For the first-stage stimulation, the user must input the following parameters directly into LabVIEW. These parameters determine the stimulation characteristics and allow for control over the specific stimulation instructions the program receives from the user. The values chosen below were empirically determined to yield the optimal results, both minimizing overall surgery time while still covering the full spectrum of voltages that could possibly initiate an action potential.

Table 4: Stage 1 Parameter values

Parameter

\begin{tabular}{|c|c|c|}
\hline Parameter & Explanation & Value \\
\hline Sampling Rate & $\begin{array}{l}\text { How many values per second } \\
\text { will be recorded }\end{array}$ & $20000 \mathrm{~Hz}$ \\
\hline Initial Voltage & $\begin{array}{c}\text { First voltage at which the } \\
\text { nerve is stimulated }\end{array}$ & $0 \mathrm{~V}$ \\
\hline Voltage Step & $\begin{array}{l}\text { Amplitude of each increase } \\
\text { in voltage }\end{array}$ & $0.5 \mathrm{~V}$ \\
\hline $\begin{array}{l}\text { Maximum Voltage } \\
\text { (Amplitude Limit) }\end{array}$ & $\begin{array}{l}\text { Max voltage, at which the } \\
\text { program will stop stimulating }\end{array}$ & $5 \mathrm{~V}$ \\
\hline $\begin{array}{l}\text { Number of Pulses Before } \\
\text { Increasing Voltage }\end{array}$ & $\begin{array}{l}\text { How many pulses will be } \\
\text { delivered at each voltage step } \\
\text { before increasing }\end{array}$ & 3 pulses \\
\hline Pulse Frequency & $\begin{array}{c}\text { Time rate at which pulses } \\
\text { will be delivered }\end{array}$ & $1 \mathrm{~Hz}$ \\
\hline Duty Cycle & $\begin{array}{c}\text { Percentage of each square } \\
\text { wave cycle that is actually } \\
\text { delivering signal to the nerve }\end{array}$ & $0.01 \%$ \\
\hline
\end{tabular}


While this stimulation is running, the operator must keep a keen eye on the oscilloscope, watching out for an action potential. A voltage between $0 \mathrm{~V}$ and $5 \mathrm{~V}$ will result in an action potential, and this value must be recorded to use as a reference for stage two. The Initial Voltage value of the second stage will have an amplitude of $0.5 \mathrm{~V}$ less than that of stage one. This allows a small cushion of subthreshold stimulation before action potentials occur.

The values from the first stage only call for 3 pulses at each voltage step before increasing the stimulation voltage. This low-resolution current step aims only to gain a rough estimate of a starting voltage for the actual stimulation. When this is determined, the operator can bypass the lowest subthreshold voltages altogether, which saves memory in the computer so that more values can be stored and so that the stimulation protocol runs more quickly. The first stage will result in an action potential seen somewhere between $0 \mathrm{~V}$ and $5 \mathrm{~V}$. The value obtained will be used as a reference for the starting point of the stimulation protocol below. Once again, the operator must change the initial input values from before to those listed in Table (5) below: 
Table 5: Stage 2 Stimulator values

Parameter

Sampling Rate

Initial Voltage

Voltage Step

Maximum Voltage (Amplitude Limit)

Number of Pulses Before Increasing Voltage

Pulse Frequency

Duty Cycle
Value

$20000 \mathrm{~Hz}$

$0.5 \mathrm{~V}$ less than the Voltage at which the first AP was observed [V]

$0.01 \mathrm{~V}$

$10 \mathrm{~V}$

\section{5 pulses}

$1 \mathrm{~Hz}$

$0.01 \%$

The user will notice differences between the values in the above two stages beyond the obvious difference between Initial Voltages. First, the Voltage Step is much smaller in the second stage. This is attributed to the higher current resolution discussed above. Next, the Maximum Voltage is higher in stage two because the intention is to stop stimulating only when no more nerve fibers are available to be stimulated (i.e. when the nerve is maximally stimulated). This is the point at which all the fibers are stimulated. The Number of Pulses Before Increasing Voltage is also higher in stage two simply because stage one is only an approximation of the initial voltage, so fewer pulses per step are permissible.

1. Ensure that the dial settings on the DAM50 differential amplifiers are set exactly as shown below in Table (6): 
Table 6: Differential Amplifier settings.

\begin{tabular}{|cc|}
\hline \multicolumn{1}{|c|}{ Setting } & Value \\
\hline Input Select & $\mathrm{A}-\mathrm{B}$ \\
Low Filter & $1 \mathrm{~Hz}$ \\
High Filter & $10 \mathrm{kHz}$ \\
Mode & $\mathrm{AC}$ \\
Gain & 100 \\
\hline
\end{tabular}

2. Ensure that the dial settings of the voltage-controlled current source are set such that the maximum current injected into the nerve is $1 \mathrm{~mA}$.

3. Ensure that the leads of the stimulation site on the electrode are connected to the current source correctly. Each cuff lead is labeled by a color-coded bead. The distances between the stimulation site and the recording sites are important, so verification is desirable.

a. The two consecutive cuffs on the nerve electrode that make up the stimulation site should be oriented such that lead associated with the proximal cuff connects to the positive red terminal of the current source, and the lead coming off the distal end connects to the black negative end.

4. At a distance of $4 \mathrm{~mm}$ from the stimulation site, place the first (proximal) recording site:

a. The proximal cuff has a lead that should be connected to a long red adapter as shown above in Figure (23). Insertion of another material for retention may be required to ensure that the lead maintains physical contact with the adapter. 
b. The other end of this red adapter should be connected to a black wire running to slot A of the electrode adapter box. Slot A is identified by its grey coloration.

5. Place the second (distal) recording site $2 \mathrm{~mm}$ distally from the first recording site:

a. The lead to this cuff connects to another red adapter similar to that of the first recording site.

b. The red adapter, as in the preceding step, should connect to Slot A of the other electrode adapter box.

6. Wire the electrode adapter boxes, differential amplifiers, current source, and nerve cuff electrode according to the diagram below, which shows the hardwiring of the entire hardware setup inside the Faraday Cage.

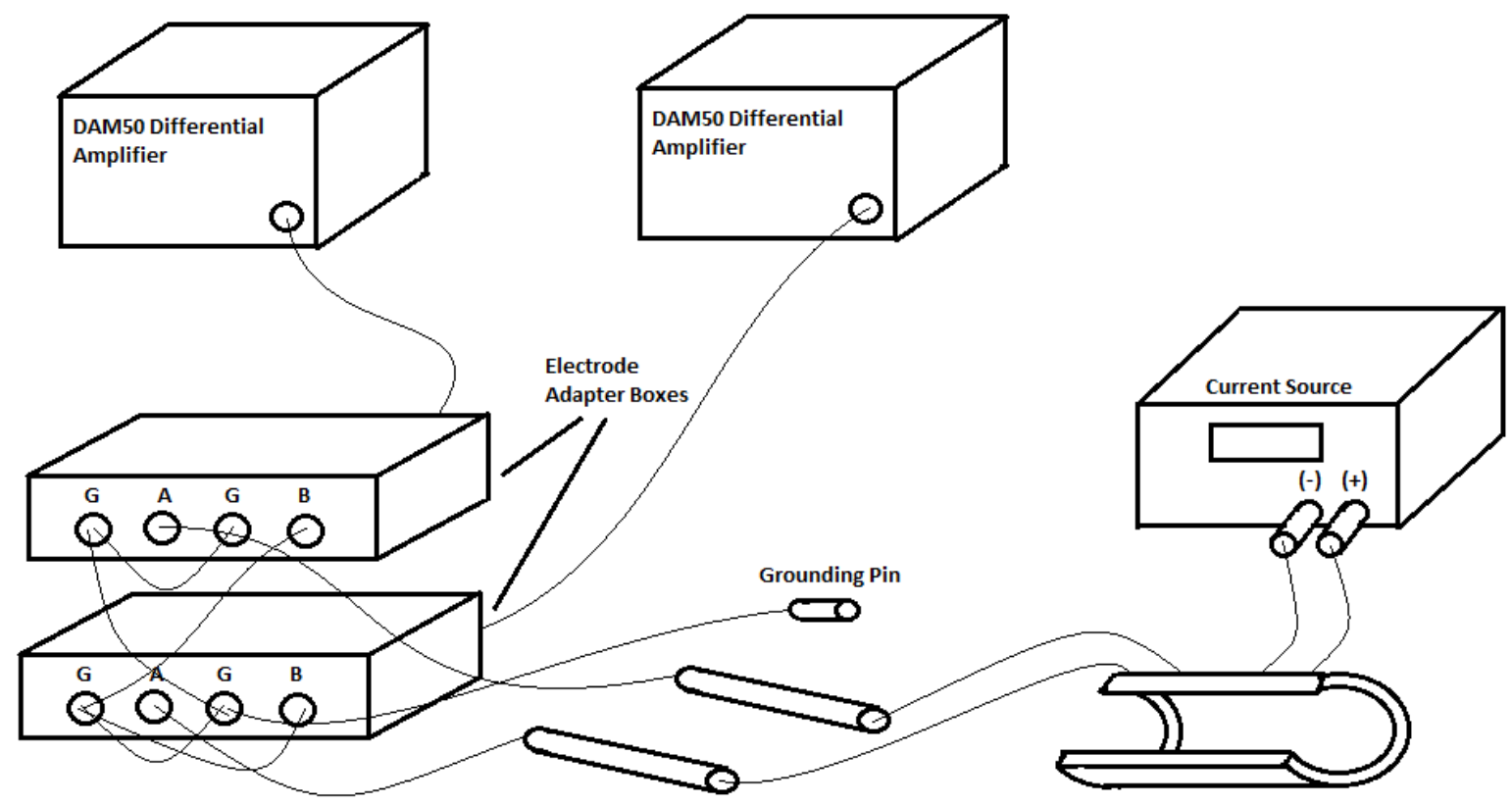

Figure 28: Schematic of differential amplifier, electrode adapter box and stimulator.

Figure (28) shows the wiring schematic for all the electronic hardware. The proximal end of the electrode is the end of the cuff that is closer to the right of the figure. One will notice that 
within the electrode adapter box, several ports are wired together. These are the Ground ports on each adapter box as well as the second Ground port on the first and the first Ground port on the second. The purpose of this interconnection scheme is to minimize the number of grounding electrodes embedded in the tissue of the animal. With the wiring method shown in the above diagram, only a single ground electrode is required in addition to the electrode cuff. This ground electrode should be placed a few millimeters away from the electrode but still within the surgery site to give an accurate local reading for ground.

7. The electrode adapter box connected to the proximal recording site should be connected, via telephone-style connector, to one of the DAM50 amplifiers. This amplifier should then be connected to the A/D converter via channel "AI0" (read: analog input zero).

8. The electrode adapter box connected to the distal recording site should be connected, via telephone-style connector, to the other of the DAM50 amplifier. This one should then be connected to the same A/D converter via channel "AI1" (read: analog input one).

9. Turn on the oscilloscopes, the differential amplifiers, and the current source. When the current source is turned on, the leg may kick.

10. The oscilloscope should show three different waveforms as flat lines.

11. Press "Begin Stimulation" using the LabVIEW stimulation software to begin the stimulation program. Do not disturb the animal during stimulation so as to avoid picking up any artifacts of motion or nerve strain when the protocol is running. 


\section{4: Termination}

With the stimulation completed and data recorded, it is time to sacrifice the specimen. The following steps are expressed as approved by IACUC, though other methods are available. Any deviation from these methods must be advisor-approved.

1. First turn off the current source.

2. Carefully remove the electrode from the nerve by sliding it out the same way it was inserted.

3. Unscrew the electrode leads from the current source. Place the electrode aside.

4. Remove the surgical tape fixing the leg to the clippers and discard.

5. Use the clippers to clip the fur on the sector of the thorax on the ribcage from the sternum to the left forelimb. The purpose of removing this fur is to make access to the chest cavity easier.

6. As before, use gauze dipped in depilatory cream to completely rid the area of fur. Wait 2 minutes before removing.

7. Use a clean piece of gauze to wipe off the hair and excess depilatory cream.

8. Gripping the skin with forceps, use surgical scissors to make a vertical incision from the bottom of the ribcage up to the level of the sternum.

9. Carefully dissect the pectoralis superficialis and reflect it out of the way, exposing the ribcage.

10. Next, dissect away the pectoralis profundus. This will allow for access to the ribcage.

11. Replace the surgical scissors with a scalpel and make an incision near the top of the ribcage, below the clavicle bone. The incision is made to access the thoracic cavity, in particular the heart. 
12. Using surgical scissors, locate the most inferior end of the sternum. There will be a small cartilaginous extension known as the xiphoid process. Directly to the left of this is the starting point of the cut. Use the surgical scissors to carefully snip through the ribcage all the way up past the sternal notch.

13. Carefully hold open the ribcage and locate the heart. The bottom left compartment of the heart is the left ventricle. Snip through it and allow blood to flow into the thoracic cavity. Once the cavity has stopped filling with blood, the animal can be considered dead. Even after termination, the nerves in the heart may still cause beating. In this case, it is recommended that the heart be excised with surgical scissors.

14. Once pronounced deceased, wrap the animal up in the dissection drape and place into a biohazard bag. Dispose of the animal in the appropriate biohazard freezer. 


\section{Chapter 9 \\ Results \& Discussion}

The surgeon and operator now have all necessary instructions to retrieve a successful nerve stimulation from the sciatic nerve of a rat. Data analysis is all that is left to complete. If signals were successfully obtained from a nerve sample, visualization of such data can be accomplished by a bar graph of the estimated fiber diameters. Data can be compiled in programs such as MATLAB, where the operator is able to not only organize the data, but can also write scripts such as those presented in Chapter 4: Group Delay. From this data, one can then take each increase in voltage response to be the addition of another neuron and then from there, graph the spectrum of voltage responses from $0 \mathrm{~V}$ up to maximal stimulation. The information that would be obtained from this would help quantify the differences in stimulation threshold between healthy animals and those with peripheral neuropathies such as that associated with diabetes.

A histogram can be obtained by plotting the action potentials incurred at different voltage steps during stimulation. Given that the current resolution is small enough, each increase is assumed to represent the recruitment of an additional neuron and each associated conduction velocity was stored. From there, the conduction velocity distribution may be divided into increments based on idealized neuron diameter. It should be noted, however, that this method of analysis is subject to error. For example, variations in conduction characteristics due to myelination are ignored. Assumptions such as this result in erroneous values such as the length scale on the horizontal axis. But the application itself can still be 
used in a clinical setting, if assumptions are minimized and a reliable relationship between conduction velocity, myelination, and fiber diameter is established.

The potential of this technology has applicability in numerous fields, such as that of neuropathic diagnosis, physical neural trauma assessment, and even potentially nerve regeneration studies. Nerve damage can often be difficult to quantify because of the difficulty in accessing the nerve as well as the symptoms most often being varying degrees of numbness and inability to carry signals. The ability to accurately quantify the maximal conduction velocity of a damaged limb compared to the contralateral healthy limb would give clinicians numerical data to assess the extent of damage. This surgery and stimulation protocol is designed to obtain optimum nerve conduction readings. They are designed to be performed by a researcher with moderate experience in animal procedures. 


\section{Bibliography}

[1] Koeppen, Bruce M.; Standon, Bruce A. Berne \& Levy Physiology $6^{\text {th }}$ ed. Mosby: Elsevier, 2008.

[2] Behse, Friedrich; Buchthal, Fritz; Carlsen, Frits. "Nerve Biopsy and Conduction Studies in Diabetic Neuropathy," Journal of Neurology, Neurosurgery, and Psychiatry, vol. 40, 1072-1082, 1977.

[3] Saladin, Kenneth S. Anatomy \& Physiology: The Unity of Form and Function, $4^{\text {th }}$ ed. George College and State University. New York: McGraw Hill Companies, 2007.

[4] Szlavik, Robert. "A Novel Method for Characterization of Peripheral Nerve Fiber Size Distribution by Group Delay," IEEE Transactions on Biomedical Engineering, vol. 55, no. 12, December 2008.

[5] Szkudelski, T. "The Mechanism of Alloxan and Streptozotocin Action in the B Cells of the Rat Pancreas," Physiol. Res. 50: 536-546, 2001.

[6] Hebel, R.; Stromberg, M.W. Anatomy of the Laboratory Rat. The Williams \& Wilkins Company, 1972.

[7] Probst, R.; Lim J.; Bird, D.; Pole, G;. Sato, A.; Claybaugh J. "Gender Differences in the Blood Volume of Conscious Sprague-Dawley Rats" Journal Am Associated Laboratory of Animal Science, vol. 45, 49-52, March 2006. 